\title{
36. INORGANIC GEOCHEMICAL COMPOSITION OF OLIGOCENE TO MIOCENE SEDIMENTS AND PRODUCTIVITY VARIATIONS IN THE WESTERN EQUATORIAL ATLANTIC: RESULTS FROM SITES 926 AND $929^{1}$
}

\author{
G.P. Weedon ${ }^{2}$ and N.J. Shackleton ${ }^{3}$
}

\begin{abstract}
Approximately 500 closely spaced pelagic sediment samples, representing the latest Miocene, middle Miocene, Oligocene/ Miocene boundary, and mid-Oligocene, were obtained from two sites on the Ceara Rise. Inductively coupled plasma emission spectroscopy was used to measure the concentrations of $\mathrm{Al}, \mathrm{Ca}, \mathrm{P}, \mathrm{K}, \mathrm{Si}, \mathrm{Ti}, \mathrm{Ba}, \mathrm{V}$, and $\mathrm{Cu}$. Normalization by $\mathrm{Al}$ was used to allow for variable carbonate dilution of the siliciclastic components. For comparison, Holocene hemipelagic silty clay samples from the Amazon Fan were also analyzed. The elemental ratios were used to infer varying sediment compositions and surface productivity.

On average, $\mathrm{K} / \mathrm{Al}$ and $\mathrm{Ti} / \mathrm{Al}$ values are similar in the latest Miocene to Holocene values, but are lower in all the older samples. This reflects the switch from kaolinite to illite-dominated clay minerals in the late Miocene, during the growth of the Amazon Fan. Average $\mathrm{Ba} / \mathrm{Al}, \mathrm{P} / \mathrm{Al}$, and $\mathrm{Si} / \mathrm{Al}$ values from the mid-Oligocene and Oligocene/Miocene boundary samples are higher than those of the middle Miocene to Holocene samples. The higher ratios suggest phases of relatively high surface productivity, which at least partly accounts for the occurrence of biosiliceous plankton microfossils in these intervals.

In the mid-Oligocene and Oligocene/Miocene boundary samples, variations in $\mathrm{Ba} / \mathrm{Al}, \mathrm{P} / \mathrm{Al}$, and $\mathrm{Si} / \mathrm{Al}$ are correlated, suggesting variable productivity over short time periods. Maximum productivity occurred during times of maximum burial/preservation of calcium carbonate. Spectral analysis reveals statistically significant regular cyclicity in all of these parameters from these intervals. The cyclicity is associated with the 40,000-yr obliquity orbital-climatic cycles. The dominance of this cyclicity, and lack of evidence for 20,000-yr cycles, suggests that short-term variations in productivity were driven by high-latitude climatic processes perhaps acting through bottom-water production.
\end{abstract}

\section{INTRODUCTION}

Drilling at five sites on the Ceara Rise during Ocean Drilling Program (ODP) Leg 154 provided a nearly complete record, at the tens of thousands of years scale, of pelagic sedimentation since the midPaleocene (Fig. 1; Curry, Shackleton, Richter, et al., 1995). The cores were collected from a depth transect of $1.5 \mathrm{~km}$ to investigate the paleoceanographic and paleoclimatic history of the equatorial Atlantic and South America. This study attempts to characterize the variations in the composition of the sediments by using inorganic elemental ratios. Four time intervals have been selected for sampling from cores from a relatively shallow site on the Rise, Site 926 (currently at a water depth of $3600 \mathrm{~m}$ ). Additionally, samples have been obtained for the oldest time period at the deepest site (Site 929 at 4360 m water depth). The samples have been analyzed for a suite of elements using inductively coupled plasma-atomic emission spectroscopy (ICPAES).

Individual $10-\mathrm{cm}^{3}$ samples were collected at $10-\mathrm{cm}$ intervals. The time intervals represented by the samples are the latest Miocene, the middle Miocene, the Oligocene/Miocene boundary and the late early Oligocene to early late Oligocene (henceforth described as "mid-Oligocene;" Table 1). Sediment compositional variations have been monitored through normalization of elemental concentrations by aluminum. This method permits the examination of changing siliciclastic mineralogy and surface productivity, although the interpretations of particular elemental ratios is not always straightforward. The sam-

${ }^{1}$ Shackleton, N.J., Curry, W.B., Richter, C., and Bralower, T.J. (Eds.), 1997. Proc. ODP, Sci. Results, 154: College Station, TX (Ocean Drilling Program).

${ }^{2}$ Department of Geology, University of Luton, Park Square, Luton, Bedfordshire LU1 3JU, United Kingdom. graham.weedon@luton.ac.uk

${ }^{3}$ Godwin Laboratory, Department of Earth Sciences, University of Cambridge, Free School Lane, Cambridge, Cambridgeshire, United Kingdom.

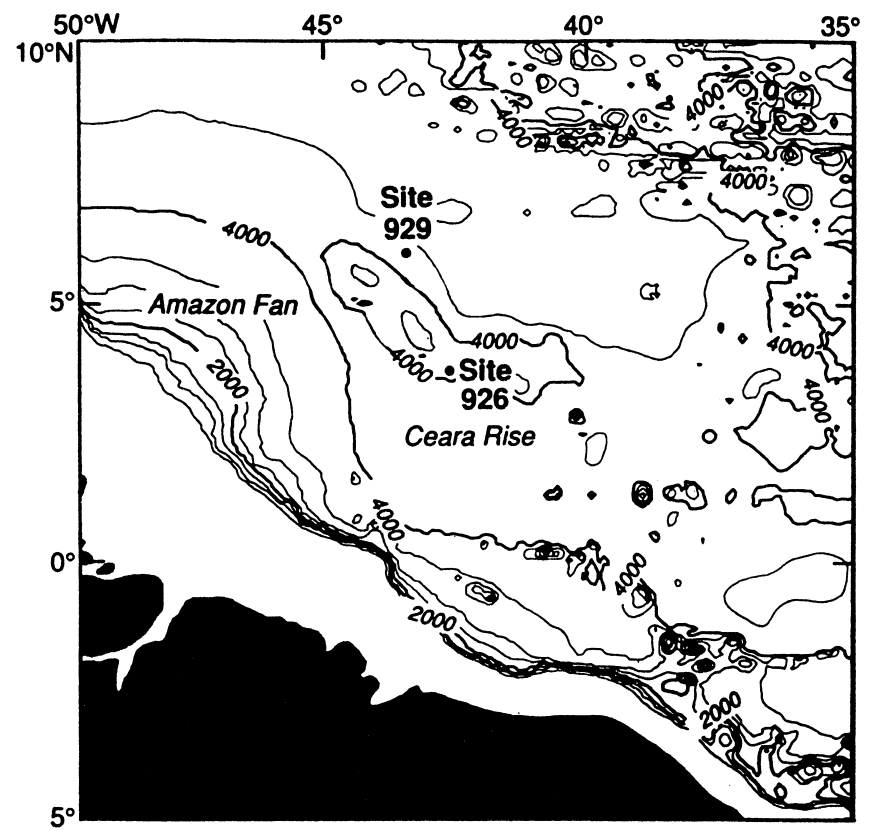

Figure 1. Location of the sites on the Ceara Rise used for sampling.

pling strategy has allowed the study of variations in these factors over time scales of both tens of millions of years and tens of thousands of years.

Since the mid-Oligocene, there have been major paleogeographical changes in the western equatorial Atlantic. In particular, the Ceara Rise has subsided as the Atlantic widened from about 6150 to 6470 
Table 1. Locations of the samples analyzed for elemental concentrations.

\begin{tabular}{|c|c|c|c|c|}
\hline Age & Biozone & Core, section, interval $(\mathrm{cm})$ & $\begin{array}{l}\text { Depth } \\
\text { (mbsf) }\end{array}$ & $\begin{array}{l}\text { Composite } \\
\text { depth (mcd) }\end{array}$ \\
\hline $\begin{array}{l}\text { Amazon Fan Samples } \\
\text { Holocene }\end{array}$ & $\mathrm{CN} 15 / \mathrm{N} 23$ & $\begin{array}{l}155-942 \mathrm{C}-2 \mathrm{H}-6,140-145 \\
155-942 \mathrm{C}-2 \mathrm{H}-7,10-14 \\
155-942 \mathrm{C}-2 \mathrm{H}-7,30-35\end{array}$ & $\begin{array}{l}13.23 \\
13.42 \\
13.63\end{array}$ & \\
\hline $\begin{array}{l}\text { Ceara Rise Samples } \\
\text { latest Miocene }\end{array}$ & $\mathrm{CN} 9 \mathrm{~b} / \mathrm{N} 17$ & $\begin{array}{l}\text { Top: } 154-926 \mathrm{~B}-17 \mathrm{H}-2,54-56 \\
\text { Base: } 154-926 \mathrm{~B}-17 \mathrm{H}-4,104-106\end{array}$ & $\begin{array}{l}151.55 \\
155.05\end{array}$ & $\begin{array}{l}171.02 \\
174.62\end{array}$ \\
\hline latest Miocene & $\mathrm{CN} 9 \mathrm{~b} / \mathrm{N} 17$ & $\begin{array}{l}\text { Top: } 154-926 \mathrm{C}-17 \mathrm{H}-6,107-109 \\
\text { Base: } 154-926 \mathrm{C}-17 \mathrm{H}-6,147-149\end{array}$ & $\begin{array}{l}161.08 \\
161.48\end{array}$ & $\begin{array}{l}180.81 \\
181.21\end{array}$ \\
\hline latest Miocene & $\mathrm{CN} 9 \mathrm{~b} / \mathrm{N} 17$ & $\begin{array}{l}\text { Top: } 154-926 \mathrm{~B}-18 \mathrm{H}-2,74-76 \\
\text { Base: } 154-926 \mathrm{~B}-18 \mathrm{H}-2,94-96\end{array}$ & $\begin{array}{l}161.25 \\
161.45\end{array}$ & $\begin{array}{l}181.29 \\
181.49\end{array}$ \\
\hline latest Miocene & CN6/N14 & $\begin{array}{l}\text { Top: } 154-926 \mathrm{~B}-25 \mathrm{H}-6,54-56 \\
\text { Base: } 154-926 \mathrm{~B}-25 \mathrm{H}-1,144-146\end{array}$ & $\begin{array}{l}226.05 \\
226.95\end{array}$ & $\begin{array}{l}252.06 \\
252.96\end{array}$ \\
\hline latest Miocene & CN5/N12-13 & $\begin{array}{l}\text { Top: } 154-926 \mathrm{~A}-26 \mathrm{H}-1,44-46 \\
\text { Base: } 154-926 \mathrm{~A}-26 \mathrm{H}-4,144-146\end{array}$ & $\begin{array}{l}232.45 \\
237.95\end{array}$ & $\begin{array}{l}260.81 \\
266.31\end{array}$ \\
\hline Miocene/Oligocene & $\mathrm{CN} 1 / \mathrm{P} 22-\mathrm{N} 4$ & $\begin{array}{l}\text { Top: } 154-926 \mathrm{~B}-50 \mathrm{X}-1,14-16 \\
\text { Base: } 154-926 \mathrm{~B}-50 \mathrm{X}-\mathrm{CC}, 44-46\end{array}$ & $\begin{array}{l}461.55 \\
470.75\end{array}$ & $\begin{array}{l}491.36 \\
500.56\end{array}$ \\
\hline latest Oligocene & $\mathrm{CN} 1 / \mathrm{P} 22$ & $\begin{array}{l}\text { Top: } 154-926 \mathrm{~B}-51 \mathrm{X}-1 \text { 14-16 } \\
\text { Base: } 154-926 \mathrm{~B}-51 \mathrm{X}-\mathrm{CC}, 34-36\end{array}$ & $\begin{array}{l}471.15 \\
480.75\end{array}$ & $\begin{array}{l}500.96 \\
510.56\end{array}$ \\
\hline late Oligocene & CP19a/P21a & $\begin{array}{l}\text { Top: } 154-926 \mathrm{~B}-64 \mathrm{X}-1,4-6 \\
\text { Base: } 154-926 \mathrm{~B}-64 \mathrm{X}-\mathrm{CC}, 24-26\end{array}$ & $\begin{array}{l}596.65 \\
606.05\end{array}$ & $\begin{array}{l}626.36 \\
635.96\end{array}$ \\
\hline early Oligocene & CP18/P19-20 & $\begin{array}{l}\text { Top: } 154-929 \mathrm{~A}-47 \mathrm{X}-1,0-2 \\
\text { Base: } 154-929 \mathrm{~A}-47 \mathrm{X}-\mathrm{CC}, 30-32\end{array}$ & $\begin{array}{l}436.11 \\
445.81\end{array}$ & $\begin{array}{l}451.53 \\
461.23\end{array}$ \\
\hline
\end{tabular}

Note: All the Ceara Rise samples were collected in continuous runs at $10-\mathrm{cm}$ intervals. Nannofossil and planktonic foraminifer biozone designations are also provided.

$\mathrm{km}$ along the line of the equator (Smith et al., 1994). Sea level has varied substantially (Haq et al., 1987) and the carbonate compensation depth has changed, whereas the extensive Miocene epeiric seas of South America were replaced by terrestrial conditions. The growth of the Amazon Fan in the latest Miocene was perhaps associated with rapid uplift of the Andes (Castro et al., 1978; Benjamin et al., 1987; Rasanen et al., 1995; Webb, 1995). All these events may have affected the sediment compositions examined here.

\section{ANALYTICAL METHODS}

The elemental concentrations of $\mathrm{Al}, \mathrm{Ba}, \mathrm{Ca}, \mathrm{Cu}, \mathrm{K}, \mathrm{P}, \mathrm{Si}$, Ti, and $\mathrm{V}$ were determined using a Perkin Elmer Plasma 40 ICP-AES. About $5 \mathrm{~g}$ of sediment was crushed using a pestle and mortar and then airdried at $105^{\circ} \mathrm{C}$. Lithium metaborate $(1.25 \mathrm{~g})$ was mixed with $0.25 \mathrm{~g}$ of sample powder in a graphite crucible and placed in a muffle furnace at $1050^{\circ} \mathrm{C}$ for $20 \mathrm{~min}$. The melt was tipped into $3.5 \%$ nitric acid and mixed until complete dissolution had occurred (after 30 min to 1 $\mathrm{hr}$ ). The solution was filtered to remove any carbon particles from the crucible and made up to $250 \mu \mathrm{L}$ with distilled water. Calibration was based on four international rock standards (CANMET LKSD1 through 4). Background correction was applied during analysis to minimize potential matrix effects. A monitor solution was run after every six unknown solutions to allow correction for drift. Accuracy and precision is presented for each element in Table 2 . The results are expressed as elemental (not oxide) concentrations in percentages for the major elements and in micrograms per gram, or parts per million, for the minors (Table 3). Copper and vanadium values were detectable, but below the limits of quantitation (i.e., ten times the standard deviation of the blank: $100 \mu \mathrm{g} / \mathrm{g}$ for $\mathrm{Cu}$ and $250 \mu \mathrm{g} / \mathrm{g}$ for $\mathrm{V}$ ) in virtually all samples. Because these elements were detectable, but not accurately quantifiable, the results for $\mathrm{Cu}$ and $\mathrm{V}$ are presented in Table 3 , but have not been illustrated. A correlation matrix for the elemental concentrations is presented in Table 4.

Calcium carbonate determinations were performed on splits of 37 samples from Core 154-926B-17H at the University of Stockholm by J. Backman. A 30-mg sample was dried and crushed using a Retch mill grinder. Analysis was performed using a UIC $\mathrm{CO}_{2}$ coulometer and the results are listed in Table 5.

\section{ELEMENTAL RATIOS AS MONITORS OF SEDIMENT COMPOSITION AND ENVIRONMENTAL PARAMETERS}

The pelagic sediments of the Ceara Rise consist of nannofossil oozes and chalks that alternate, on a decimeter-to-meter scale, with clayey nannofossil oozes and chalks. The causes of these variations in carbonate contents are unclear, but are at least partly related to carbonate dissolution as revealed by foraminifer preservation (Pearson et al., this volume). Whatever processes were responsible for the carbonate fluctuations, their regularity is diagnostic of indirect control by orbital-climatic cycles (Weedon, et al., this volume).

To allow for the substantial variations in carbonate content, normalization of the element concentrations by aluminum has been employed (Shimmield and Mowbray, 1991; Norry et al., 1994). This procedure relies on the presence of aluminum in the non-carbonate (principally clay mineral) fraction. Although it is not suggested that the average content of aluminum in the non-carbonate fraction was constant, it can reasonably be assumed that it varied by a much smaller factor than bulk carbonate contents. Support for the assertion that aluminum is associated with the siliciclastic fraction of these sediments comes from the very high correlation (Table 4) with $\mathrm{Si}$, Ti, and $\mathrm{K}$ and the inverse correlation with $\mathrm{Ca}$ as illustrated in Fig. 2.

Since the composition of the siliciclastic fraction of the sediments is the main subject of this study, samples were analyzed from the Holocene Amazon Fan. The Amazon Fan is not active during the current sea level high-stand. Therefore, three samples that date from the last glacial maximum were obtained from hemipelagic silty clay on a channel levee from Site 942, ODP Leg 155 (Flood, Piper, Klaus, et al., 1995). The current water depth of this site is $3350 \mathrm{~m}$ so it would have been very close to the carbonate compensation depth at $18 \mathrm{ka}$. The Ceara Rise and Amazon Fan currently underlie a low productivity zone; the fan samples provide an indication of near-Holocene fine-grained siliciclastic sediment compositions (Table 3). 
Table 2. Accuracy and precision based on replicate analyses of CANMET lake sediment standard LKSD 4.

\begin{tabular}{|c|c|c|c|c|c|c|}
\hline Element & $\begin{array}{c}\text { No. repeat } \\
\text { analyses }\end{array}$ & $\begin{array}{l}\text { Mean of } \\
\text { analyses }\end{array}$ & SD & LKSD 4 & $\begin{array}{c}\text { Accuracy } \\
(\%)\end{array}$ & $\begin{array}{c}\text { Precision } \\
(\%)\end{array}$ \\
\hline $\mathrm{Al}$ & 20 & $6.46 \%$ & $0.136 \%$ & $6.40 \%$ & 100.9 & 2.1 \\
\hline $\mathrm{Ca}$ & 20 & $2.89 \%$ & $0.069 \%$ & $2.86 \%$ & 101.3 & 2.4 \\
\hline $\mathrm{P}$ & 20 & $0.088 \%$ & $0.0028 \%$ & $0.087 \%$ & 100.7 & 3.2 \\
\hline $\mathrm{K}$ & 20 & $1.36 \%$ & $0.064 \%$ & $1.33 \%$ & 102.4 & 4.7 \\
\hline $\mathrm{Si}$ & 20 & $27.5 \%$ & $0.74 \%$ & $27.5 \%$ & 100.0 & 2.7 \\
\hline $\mathrm{Ti}$ & 20 & $0.487 \%$ & $0.011 \%$ & $0.480 \%$ & 101.4 & 2.2 \\
\hline $\mathrm{Ba}$ & 20 & $2018 \mu \mathrm{g} / \mathrm{g}$ & $65 \mu \mathrm{g} / \mathrm{g}$ & $2000 \mu \mathrm{g} / \mathrm{g}$ & 100.9 & 3.2 \\
\hline V & 6 & $106 \mu \mathrm{g} / \mathrm{g}$ & $10 \mu \mathrm{g} / \mathrm{g}$ & $106 \mu \mathrm{g} / \mathrm{g}$ & 100.0 & 9.4 \\
\hline $\mathrm{Cu}$ & 19 & $63 \mu \mathrm{g} / \mathrm{g}$ & $7 \mu \mathrm{g} / \mathrm{g}$ & $65 \mu \mathrm{g} / \mathrm{g}$ & 96.9 & 11.1 \\
\hline
\end{tabular}

Notes: Accuracy is defined as $100 \%$ times the mean of the repeat analyses divided by the expected value. Precision is defined as $100 \%$ times the best estimate standard deviation $\left(\sigma_{\mathrm{n}-1}\right)$ divided by the mean of the repeats. $\mathrm{SD}=$ standard deviation.

The elements chosen for study are inferred to act, once normalized by aluminum, as proxies for sediment composition and paleoceanographic parameters following the strategy of, for example, Shimmield and Mowbray (1991). Many factors can influence elemental ratios, so each element is considered in turn.

In pelagic sediments, potassium resides almost exclusively in clay minerals and in particular within illite (Van Buchem et al., 1994). Absolute potassium concentrations are strongly correlated with $\mathrm{Al}$ and Ti (Table 4; Fig. 2). Titanium resides within heavy minerals such as ilmenite and rutile and within clay minerals. Consequently, the Ti/Al ratio provides an indication of the relative proportions of heavy minerals in the siliciclastic fraction and variations in clay mineralogy (Schmitz, 1989; Shimmield and Mowbray, 1991). The association of $\mathrm{Ti}$ with the siliciclastic components explains the high correlation with $\mathrm{Al}, \mathrm{Si}$, and $\mathrm{K}$, and the inverse correlation with $\mathrm{Ca}$ in Table 4 .

Barium is deposited in pelagic sediments as biogenic barites, and is incorporated structurally within biogenic calcite and in clay minerals (Dymond et al., 1992; Lea and Spero, 1994; Gingele and Dahmke, 1994). Consequently, high barium concentrations can follow other productivity proxies. As an example, $\mathrm{Ba} / \mathrm{Al}$ values from recent sediments were used to monitor productivity variations over tens of thousands of years off the coast of Oman (Shimmield and Mowbray, 1991; Weedon and Shimmield, 1991). Since barium occurs in siliciclastic minerals, however, an estimation of detrital $\mathrm{Ba} / \mathrm{Al}$ values is needed. In the three Amazon Fan samples analyzed, the ratios ranged from 0.0032 to 0.0034 , whereas average values for siliciclastic minerals are 0.005 to 0.01 (Dymond et al., 1992). Sulfate reduction can cause barite dissolution (Brumsack and Gieskes, 1983). At Sites 926 and 929 , pore-water sulfate concentrations are moderately reduced compared to seawater (Curry, Shackleton, Richter, et al., 1995). Because some sulfate reduction has occurred in Ceara Rise sediments, the $\mathrm{Ba} / \mathrm{Al}$ ratios measured may have been lowered during diagenesis. Therefore, in this study, values that are higher than detrital values (i.e., above 0.01) have been taken as indicative of enhanced productivity. Using all the samples examined here, Ba concentrations are weakly negatively correlated with the siliciclastic elements $\mathrm{Al}, \mathrm{K}$, and $\mathrm{Ti}$ (Table 4). This inverse relationship suggests that, on average, most Ba in Ceara Rise sediments is not associated with clays

As phosphorus is a limiting nutrient in the oceans and is associated with organic matter, phosphorus concentrations in sediments have been used to monitor changing surface productivity (Filippelli and Delaney, 1994). Pauses in sedimentation and hiatuses can lead to phosphorus remobilization and authigenic concentration at discrete horizons. However, at Site 926, sedimentation rates were relatively high and there is no evidence of hiatuses (Curry, Shackleton, Richter, et al., 1995). Similarly, dysoxic and anoxic bottom waters can lead to increased recycling of phosphorus into the water column, which decreases the proportion buried (Van Cappellen and Ingall, 1994). Yet on the Ceara Rise, there is no evidence for laminated sediments; all of the pelagic sediments are thoroughly bioturbated, and the organic carbon concentrations are consistently very low $(<0.25 \%$; Curry, Shackleton, Richter, et al., 1995). Some P is incorporated in detrital minerals, and here it is weakly positively correlated with $\mathrm{Al}, \mathrm{Si}, \mathrm{Ti}$, and $\mathrm{K}$ (Table 4). Hence, as for barium, a comparison with the Holocene Amazon Fan samples has been used to assess whether particular values indicate enhanced productivity

Silicon is strongly correlated with the siliciclastic elements $\mathrm{Al}, \mathrm{Ti}$, and $\mathrm{K}$, reflecting its presence in clay minerals and, probably minor, quartz. Potentially, though, the Si/Al value could depend partly on concentrations of biogenic opal (Lyle et al., 1988; Charles et al., 1991). Indeed, diatoms and radiolaria were observed forming significant proportions of the lower Miocene and lower Oligocene sediment, although no chert was encountered (Curry, Shackleton, Richter, et al., 1995). Although the occurrence of biogenic silica can be indicative of high productivity, the factors that control the concentrations in ancient sediments are complex (Archer et al., 1993). For example, silica dissolution in carbonate-rich sediments during burial is a function of absolute silica concentrations. Therefore, in the case of sediments that received a constant flux of biogenic silica, but variable carbonate, $\mathrm{Si} / \mathrm{Al}$ ratios, or silica expressed on a carbonate-free basis, would yield a spurious variation related to the effect of carbonate concentration upon silica dissolution (Archer et al., 1993). This would produce a covariation of carbonate and $\mathrm{Si} / \mathrm{Al}$ values. In such situations other proxies such as $\mathrm{Ba} / \mathrm{Al}$ and $\mathrm{P} / \mathrm{Al}$ are needed to check that the $\mathrm{Si} / \mathrm{Al}$ variations include a productivity component.

Vanadium has been observed at a concentration well above that of "average shale" in strata associated with reduced bottom-water oxygenation and under areas of high productivity (Brumsack, 1986; Arthur et al., 1989). Elevated V deposition appears to be associated with binding to organic matter. However, in all the samples analyzed, vanadium occurs at levels below the limit of quantitation. Copper is also associated with sediments containing high organic carbon concentrations associated with areas of high productivity (Arthur et al., 1989). As for vanadium, concentrations are always below the limit of quantitation. For both of these elements, the low concentrations are probably connected to the very low total organic carbon contents in the cores examined, caused by thorough ventilation during bioturbation. The $\mathrm{V}$ and $\mathrm{Cu}$ concentrations are recorded in Table 3, but since these elements occur in detectable, but not accurately quantifiable levels, they are not illustrated and are not discussed further.

Part of the scatter observed in the plot of Ca vs. Al for all samples (Fig. 2) might reflect the presence of calcium within clays and long term changes in clay mineralogy. However, there is evidence for a linear relationship between $\mathrm{Ca} / \mathrm{Al}$ and carbonate divided by non-carbonate in Core 154-926B-17H, and when plotted stratigraphically, this ratio covaries with $\% \mathrm{CaCO}_{3}$ (Fig. 3). Hence, we have used plots of $\mathrm{Ca} / \mathrm{Al}$ as indicative of carbonate contents over short stratigraphic intervals.

\section{RESULTS \\ Long-Term Changes in Elemental Ratios}

To gauge the long-term changes in the elemental ratios, all of the results are plotted for Site 926 in Figure 4. For comparison, the vertical dashed lines indicate the ratios determined for the Holocene Amazon Fan samples.

The estimated average carbonate contents since the mid-Oligocene show small changes over millions of years in terms of variance and average values. This may be partly related to changing dissolution on the seafloor associated with the depth of the lysocline and/or to changing dilution by the siliciclastic flux. Average barium ratios in the latest Miocene and middle Miocene are essentially identical to the Holocene Amazon Fan hemipelagic silty clay. At the Oligocene/Miocene boundary and in the mid-Oligocene, the ratios are considerably higher, probably because of much higher productivity. This is confirmed by the very similar pattern found for phosphorus 
Table 3. Elemental concentrations for samples from the Ceara Rise and Amazon Fan.

\begin{tabular}{|c|c|c|c|c|c|c|c|c|c|c|c|}
\hline $\begin{array}{l}\text { Core, section, } \\
\text { interval }(\mathrm{cm})\end{array}$ & $\begin{array}{l}\text { Depth } \\
\text { (mbsf) }\end{array}$ & $\begin{array}{l}\text { Depth } \\
\text { (mcd) }\end{array}$ & $\begin{array}{c}\mathrm{Al} \\
(\%)\end{array}$ & $\begin{array}{l}\mathrm{Ca} \\
(\%)\end{array}$ & $\begin{array}{c}\mathrm{P} \\
(\%)\end{array}$ & $\begin{array}{c}\mathrm{K} \\
(\%)\end{array}$ & $\begin{array}{c}\mathrm{Si} \\
(\%)\end{array}$ & $\begin{array}{c}\mathrm{Ti} \\
(\%)\end{array}$ & $\begin{array}{c}\mathrm{Ba} \\
(\mathrm{ppm})\end{array}$ & $\begin{array}{c}\mathrm{V} \\
(\mathrm{ppm})\end{array}$ & $\begin{array}{c}\mathrm{Cu} \\
(\mathrm{ppm})\end{array}$ \\
\hline $155-942 C-$ & & & & & & & & & & & \\
\hline $2 \mathrm{H}-6,140-145$ & 13.23 & 13.23 & 10.48 & 1.81 & 0.059 & 2.40 & 23.6 & 0.561 & 431 & 151 & 56 \\
\hline $2 \mathrm{H}-7,10-14$ & 13.42 & 13.42 & 10.13 & 1.29 & 0.062 & 2.44 & 24.5 & 0.583 & 439 & 148 & 40 \\
\hline $2 \mathrm{H}-7,30-35$ & 13.63 & 13.63 & 9.84 & 2.25 & 0.098 & 2.24 & 22.4 & 0.551 & 400 & 151 & 57 \\
\hline 154-926B- & & & & & & & & & & & \\
\hline $17 \mathrm{H}-2,54-56$ & 151.55 & 171.02 & 2.39 & 21.29 & 0.028 & 0.59 & 5.1 & 0.132 & 185 & 105 & 23 \\
\hline $17 \mathrm{H}-2,64-66$ & 151.65 & 171.12 & 2.34 & 14.97 & 0.026 & 0.69 & 5.6 & 0.157 & 146 & 20 & 23 \\
\hline $17 \mathrm{H}-2,74-76$ & 151.75 & 171.22 & 4.90 & 17.83 & 0.036 & 1.30 & 10.8 & 0.277 & 297 & 149 & 37 \\
\hline $17 \mathrm{H}-2,84-86$ & 151.85 & 171.32 & 5.28 & 16.34 & 0.034 & 1.21 & 11.2 & 0.307 & 294 & 132 & 33 \\
\hline $17 \mathrm{H}-2,94-96$ & 151.95 & 171.42 & 5.26 & 16.84 & 0.035 & 1.33 & 11.3 & 0.297 & 278 & 118 & 12 \\
\hline $17 \mathrm{H}-2,104-106$ & 152.05 & 171.52 & 3.26 & 20.54 & 0.025 & 0.74 & 6.8 & 0.176 & 196 & 83 & 59 \\
\hline $17 \mathrm{H}-2,114-116$ & 152.15 & 171.62 & 3.93 & 19.29 & 0.035 & 0.95 & 8.3 & 0.212 & 215 & 113 & 37 \\
\hline $17 \mathrm{H}-2,124-126$ & 152.25 & 171.72 & 4.05 & 16.85 & 0.026 & 0.98 & 8.5 & 0.219 & 194 & 81 & 37 \\
\hline $17 \mathrm{H}-2,134-136$ & 152.35 & 171.82 & 5.66 & 23.81 & 0.034 & 1.04 & 11.0 & 0.285 & 143 & 40 & 26 \\
\hline $17 \mathrm{H}-2,144-146$ & 152.45 & 171.92 & 3.81 & 23.81 & 0.029 & 1.47 & 7.6 & 0.250 & 188 & 57 & 26 \\
\hline $17 \mathrm{H}-3,4-6$ & 152.55 & 172.02 & 2.87 & 19.96 & 0.026 & 0.85 & 5.8 & 0.146 & 159 & 49 & 19 \\
\hline $17 \mathrm{H}-3,14-16$ & 152.65 & 172.12 & 3.12 & 20.30 & 0.035 & 0.78 & 6.8 & 0.179 & 212 & 80 & 6 \\
\hline $17 \mathrm{H}-3,24-26$ & 152.75 & 172.22 & 3.75 & 20.10 & 0.036 & 0.99 & 8.6 & 0.200 & 218 & 79 & 18 \\
\hline $17 \mathrm{H}-3,34-36$ & 152.85 & 172.32 & 4.88 & 16.22 & 0.032 & 1.27 & $\begin{array}{l}0.0 \\
11.0\end{array}$ & 0.274 & 252 & 76 & 7 \\
\hline $17 \mathrm{H}-3,44-46$ & 152.95 & 172.42 & 5.17 & 16.30 & 0.030 & 1.14 & 11.0 & 0.224 & 245 & 89 & 16 \\
\hline $17 \mathrm{H}-3,54-56$ & 153.05 & 172.52 & 4.29 & 18.79 & 0.032 & 1.16 & 9.3 & 0.242 & 239 & 21 & 16 \\
\hline $17 \mathrm{H}-3,64-66$ & 153.15 & 172.62 & 3.64 & 20.24 & 0.030 & 0.95 & 7.8 & 0.194 & 203 & 64 & 13 \\
\hline $17 \mathrm{H}-3,74-76$ & 153.25 & 172.72 & 3.26 & 20.67 & 0.032 & 0.82 & 7.3 & 0.174 & 172 & 69 & 14 \\
\hline $17 \mathrm{H}-3,84-86$ & 153.35 & 172.82 & 3.56 & 20.98 & 0.043 & 0.64 & 7.3 & 0.176 & 172 & 57 & 27 \\
\hline $17 \mathrm{H}-3,94-96$ & 153.45 & 172.92 & 2.97 & 21.53 & 0.025 & 0.78 & 6.3 & 0.190 & 213 & 64 & 14 \\
\hline $17 \mathrm{H}-3,104-106$ & 153.55 & 173.02 & 3.01 & 22.45 & 0.029 & 1.22 & 7.0 & 0.198 & 148 & 57 & 23 \\
\hline $17 \mathrm{H}-3,114-116$ & 153.65 & 173.12 & 3.99 & 18.65 & 0.030 & 1.45 & 8.5 & 0.199 & 185 & 69 & 38 \\
\hline $17 \mathrm{H}-3,124-126$ & 153.75 & 173.22 & 4.47 & 19.15 & 0.028 & 0.96 & 9.2 & 0.159 & 199 & 36 & 27 \\
\hline $17 \mathrm{H}-3,134-136$ & 153.85 & 173.32 & 5.66 & 17.49 & 0.039 & 0.83 & 11.3 & 0.134 & 248 & 83 & 24 \\
\hline $17 \mathrm{H}-3,144-146$ & 153.95 & 173.42 & 4.17 & 19.28 & 0.032 & $\begin{array}{l}.03 \\
1.09\end{array}$ & 8.3 & 0.169 & 200 & 63 & 31 \\
\hline $17 \mathrm{H}-4,4-6$ & 154.05 & 173.52 & 5.26 & 15.71 & 0.039 & 1.71 & 11.3 & 0.269 & 234 & 88 & 65 \\
\hline $17 \mathrm{H}-4,14-16$ & 154.15 & 173.62 & 3.58 & 20.31 & 0.033 & 0.97 & 7.8 & 0.199 & 183 & 14 & 36 \\
\hline $17 \mathrm{H}-4,24-26$ & 154.25 & 173.72 & 2.65 & 18.99 & 0.024 & 0.80 & 5.6 & 0.135 & 139 & 27 & 26 \\
\hline $17 \mathrm{H}-4,34-36$ & 154.35 & 173.82 & 3.99 & 17.81 & 0.032 & 1.16 & 8.7 & 0.214 & 193 & 43 & 33 \\
\hline $17 \mathrm{H}-4,44-46$ & 154.45 & 173.92 & 6.05 & 14.97 & 0.031 & 1.44 & 13.1 & 0.290 & 271 & 73 & 24 \\
\hline $17 \mathrm{H}-4,54-56$ & 154.55 & 174.02 & 6.77 & 13.72 & 0.042 & $\begin{array}{l}1.74 \\
1.79\end{array}$ & 14.3 & 0.360 & 278 & 72 & 26 \\
\hline $17 \mathrm{H}-4,64-66$ & 154.65 & 174.12 & 6.47 & 13.98 & 0.036 & 1.51 & 13.5 & 0.333 & 279 & 65 & 23 \\
\hline $17 \mathrm{H}-4,74-76$ & 154.75 & 174.22 & 4.65 & 16.85 & 0.028 & 1.40 & 9.7 & 0.243 & 215 & 99 & 30 \\
\hline $17 \mathrm{H}-4,84-86$ & 154.85 & 174.32 & 4.77 & 16.56 & 0.034 & 1.38 & 9.6 & 0.239 & 221 & 77 & 26 \\
\hline $17 \mathrm{H}-4,94-96$ & 154.95 & 174.42 & 4.98 & 15.32 & 0.036 & 1.49 & 10.3 & 0.249 & 208 & 68 & 28 \\
\hline $17 \mathrm{H}-4,104-106$ & 155.05 & 174.52 & 5.51 & 14.60 & 0.033 & 1.58 & 11.3 & 0.278 & 223 & 108 & 30 \\
\hline $17 \mathrm{H}-4,114-116$ & 155.15 & 174.62 & 3.43 & 17.85 & 0.031 & 1.01 & 6.9 & 0.171 & 153 & 74 & 26 \\
\hline $154-926 \mathrm{C}-$ & & & & & & & & & & & \\
\hline $17 \mathrm{H}-6,107-109$ & 161.08 & 180.81 & 3.34 & 18.59 & 0.040 & 0.79 & 6.9 & 0.164 & 179 & 81 & 25 \\
\hline $17 \mathrm{H}-6,117-119$ & 161.18 & 180.91 & 4.97 & 17.04 & 0.033 & 1.19 & 10.9 & 0.242 & 243 & 118 & 16 \\
\hline $17 \mathrm{H}-6,127-129$ & 161.28 & 181.01 & 6.20 & 15.43 & 0.040 & 1.39 & 12.7 & 0.340 & 268 & 134 & 21 \\
\hline $17 \mathrm{H}-6,137-139$ & 161.38 & 181.11 & 6.74 & 14.61 & 0.035 & 1.35 & 13.7 & 0.367 & 293 & 85 & 20 \\
\hline $17 \mathrm{H}-6,147-149$ & 161.48 & 181.21 & 4.99 & 17.25 & 0.026 & 1.06 & 9.9 & 0.234 & 235 & 53 & 19 \\
\hline 154-926B- & & & & & & & & & & & \\
\hline $18 \mathrm{H}-2,74-76$ & 161.25 & 181.29 & 4.16 & 17.36 & 0.037 & 1.02 & 8.8 & 0.207 & 204 & 86 & 10 \\
\hline $18 \mathrm{H}-2,84-86$ & 161.35 & 181.39 & 5.97 & 15.66 & 0.031 & 1.41 & $\begin{array}{l}0.0 \\
12.1\end{array}$ & 0.295 & 254 & 127 & 17 \\
\hline $18 \mathrm{H}-2,94-96$ & 161.45 & 181.49 & 6.45 & 14.45 & 0.030 & 1.44 & 13.2 & 0.323 & 251 & 86 & 19 \\
\hline $25 \mathrm{H}-1,54-56$ & 226.05 & 252.06 & 6.83 & 15.55 & 0.048 & 1.10 & 12.5 & 0.336 & 231 & 58 & 31 \\
\hline $25 \mathrm{H}-1,64-66$ & 226.15 & 252.16 & 5.16 & 21.07 & 0.035 & 0.75 & 8.6 & 0.233 & 221 & 82 & 23 \\
\hline $25 \mathrm{H}-1,74-76$ & 226.25 & 252.26 & 7.80 & 15.14 & 0.043 & 1.20 & 13.4 & 0.371 & 240 & 193 & 33 \\
\hline $25 \mathrm{H}-1,84-86$ & 226.35 & 252.36 & 5.79 & 19.84 & 0.036 & 0.80 & 9.8 & 0.265 & 219 & 216 & 40 \\
\hline $25 \mathrm{H}-1,94-96$ & 226.45 & 252.46 & 8.19 & 12.60 & 0.044 & 1.41 & 15.0 & 0.430 & 266 & 106 & 27 \\
\hline $25 \mathrm{H}-1,104-106$ & 226.55 & 252.56 & 5.19 & 19.74 & 0.041 & 0.80 & 9.1 & 0.239 & 204 & 69 & 27 \\
\hline $25 \mathrm{H}-1,114-116$ & 226.65 & 252.66 & 7.15 & 15.00 & 0.047 & 1.16 & 12.5 & 0.347 & 230 & 70 & 33 \\
\hline $25 \mathrm{H}-1,126-128$ & 226.75 & 252.76 & 7.89 & 14.03 & 0.045 & 1.21 & 13.5 & 0.364 & 230 & 61 & 30 \\
\hline $25 \mathrm{H}-1,134-136$ & 226.85 & 252.86 & 5.61 & 19.68 & 0.041 & 0.79 & 9.1 & 0.249 & 184 & 108 & 34 \\
\hline $25 \mathrm{H}-1,144-146$ & 226.95 & 252.96 & 6.16 & 17.30 & 0.037 & 1.00 & 10.6 & 0.284 & 186 & 67 & 20 \\
\hline 154-926A- & & & & & & & & & & & \\
\hline $26 \mathrm{H}-1,44-46$ & 232.45 & 260.81 & 6.52 & 24.12 & 0.035 & 0.84 & 10.1 & 0.263 & 240 & 71 & 22 \\
\hline $26 \mathrm{H}-1,54-56$ & 232.55 & 260.9 & 7.61 & 13.46 & 0.052 & 1.03 & 12.9 & 0.337 & 249 & 99 & 37 \\
\hline $26 \mathrm{H}-1,64-66$ & 232.65 & 261.01 & 8.37 & 18.62 & 0.049 & 1.17 & 13.3 & 0.343 & 273 & 96 & 20 \\
\hline $26 \mathrm{H}-1,74-76$ & 232.75 & 261.11 & 6.07 & 24.41 & 0.041 & 0.92 & 10.0 & 0.268 & 198 & 75 & 33 \\
\hline $26 \mathrm{H}-1,84-86$ & 232.85 & 261.21 & 5.17 & 26.80 & 0.035 & 0.81 & 8.8 & 0.224 & 174 & 28 & 26 \\
\hline $26 \mathrm{H}-1,94-96$ & 232.95 & 261.31 & 6.82 & 22.72 & 0.031 & 0.95 & 11.9 & 0.303 & 276 & 38 & 73 \\
\hline $26 \mathrm{H}-1,104-106$ & 233.05 & 261.41 & 5.77 & 26.70 & 0.024 & 0.74 & 9.3 & 0.240 & 258 & 61 & 37 \\
\hline $26 \mathrm{H}-1,114-116$ & 233.15 & 261.51 & 5.02 & 26.70 & 0.029 & 0.78 & 8.5 & 0.211 & 223 & 44 & 93 \\
\hline $26 \mathrm{H}-1,124-126$ & 233.25 & 261.61 & 7.19 & 22.04 & 0.047 & 1.13 & 12.5 & 0.320 & 255 & 51 & 35 \\
\hline $26 \mathrm{H}-1,134-136$ & 233.35 & 261.71 & 5.11 & 26.29 & 0.033 & 0.83 & 9.0 & 0.230 & 228 & 27 & 27 \\
\hline $26 \mathrm{H}-1,144-146$ & 233.45 & 261.81 & 4.32 & 30.60 & 0.036 & 0.64 & 6.8 & 0.177 & 160 & 19 & 20 \\
\hline $26 \mathrm{H}-2,4-6$ & 233.55 & 261.91 & 8.96 & 12.25 & 0.053 & 1.19 & 17.1 & 0.522 & 308 & 142 & 37 \\
\hline $26 \mathrm{H}-2,14-16$ & 233.6 & 262. & 5.77 & 19.9 & 0.0 & 0.91 & 10.6 & 0.2 & 243 & 91 & 25 \\
\hline $26 \mathrm{H}-2,24-26$ & 233.75 & 262.11 & 7.38 & 15.44 & 0.049 & 1.12 & 13.7 & 0.359 & 232 & 110 & 48 \\
\hline $26 \mathrm{H}-2,34-36$ & 233.85 & 262.21 & 4.55 & 21.82 & 0.036 & 0.73 & 8.4 & 0.229 & 159 & 117 & 23 \\
\hline $26 \mathrm{H}-2,44-46$ & 233.95 & 262.31 & 3.51 & 22.97 & 0.033 & 0.47 & 6.8 & 0.161 & 126 & 20 & 21 \\
\hline $26 \mathrm{H}-2,54-56$ & 234.05 & 262.41 & 8.32 & 17.15 & 0.052 & 1.37 & 16.3 & 0.409 & 298 & 94 & 23 \\
\hline $26 \mathrm{H}-2,64-66$ & 234.15 & 262. & 3.59 & 22.6 & 0.03 & 0.62 & 6.4 & 0.1 & 190 & 39 & 24 \\
\hline $26 \mathrm{H}-2,74-76$ & 234.25 & 262.61 & 4.28 & 23.39 & 0.042 & 0.77 & 8.5 & 0.207 & 194 & 61 & 34 \\
\hline $26 \mathrm{H}-2,84-86$ & 234.35 & 262.71 & 4.93 & 22.26 & 0.043 & 0.82 & 8.9 & 0.245 & 166 & 69 & 34 \\
\hline $26 \mathrm{H}-2,94-96$ & 234.45 & 262.81 & 6.51 & 20.46 & 0.040 & 0.98 & 11.7 & 0.303 & 271 & 61 & 20 \\
\hline $26 \mathrm{H}-2,104-106$ & 234.55 & 262.91 & 8.94 & 16.42 & 0.050 & 1.26 & 16.6 & 0.433 & 355 & 104 & 76 \\
\hline
\end{tabular}


Table 3 (continued).

\begin{tabular}{|c|c|c|c|c|c|c|c|}
\hline $\begin{array}{l}\text { Core, section, } \\
\text { interval }(\mathrm{cm})\end{array}$ & $\begin{array}{l}\text { Depth } \\
\text { (mbsf) }\end{array}$ & $\begin{array}{l}\text { Depth } \\
\text { (mcd) }\end{array}$ & $\begin{array}{c}\mathrm{Al} \\
(\%)\end{array}$ & $\begin{array}{c}\mathrm{Ca} \\
(\%)\end{array}$ & $\begin{array}{c}\mathrm{P} \\
(\%)\end{array}$ & $\begin{array}{c}\mathrm{K} \\
(\%)\end{array}$ & $\begin{array}{l}\mathrm{Si} \\
(\%)\end{array}$ \\
\hline $26 \mathrm{H}-2,124-126$ & 234.75 & 263.11 & 9.30 & 15.80 & 0.056 & 1.48 & 17.7 \\
\hline $26 \mathrm{H}-2,134-136$ & 234.85 & 263.21 & 6.16 & 17.42 & 0.054 & 0.93 & 11.3 \\
\hline $26 \mathrm{H}-2,144-146$ & 234.95 & 263.31 & 9.44 & 15.64 & 0.058 & 1.63 & 18.5 \\
\hline $26 \mathrm{H}-3,4-6$ & 235.05 & 263.41 & 4.32 & 17.71 & 0.030 & 0.72 & 7.9 \\
\hline $26 \mathrm{H}-3,14-16$ & 235.15 & 263.51 & 5.05 & 17.51 & 0.030 & 0.79 & 9.3 \\
\hline $26 \mathrm{H}-3,24-26$ & 235.25 & 263.61 & 6.54 & 15.02 & 0.040 & 1.06 & 12.4 \\
\hline $26 \mathrm{H}-3,34-36$ & 235.35 & 263.71 & 4.31 & 17.66 & 0.045 & 0.80 & 8.0 \\
\hline $26 \mathrm{H}-3,44-46$ & 235.45 & 263.81 & 4.25 & 17.83 & 0.039 & 0.74 & 7.8 \\
\hline $26 \mathrm{H}-3,54-56$ & 235.55 & 263.91 & 4.95 & 20.99 & 0.035 & 0.80 & 9.2 \\
\hline $26 \mathrm{H}-3,64-66$ & 235.65 & 264.01 & 6.85 & 14.16 & 0.041 & 1.13 & 13.1 \\
\hline $26 \mathrm{H}-3,74-76$ & 235.75 & 264.11 & 4.78 & 24.11 & 0.043 & 0.87 & 9.0 \\
\hline $26 \mathrm{H}-3,84-86$ & 235.85 & 264.21 & 4.39 & 26.22 & 0.033 & 0.84 & 8.3 \\
\hline $26 \mathrm{H}-3,94-96$ & 235.95 & 264.31 & 4.83 & 24.43 & 0.031 & 0.92 & 9.6 \\
\hline $26 \mathrm{H}-3,104-106$ & 236.05 & 264.41 & 6.91 & 13.95 & 0.033 & 1.09 & 12.8 \\
\hline $26 \mathrm{H}-3,114-116$ & 236.15 & 264.51 & 6.29 & 14.90 & 0.032 & 0.95 & 11.8 \\
\hline $26 \mathrm{H}-3,124-126$ & 236.25 & 264.61 & 5.61 & 23.39 & 0.032 & 0.93 & 9.7 \\
\hline $26 \mathrm{H}-3,134-136$ & 236.35 & 264.71 & 4.73 & 17.79 & 0.026 & 0.84 & 8.2 \\
\hline $26 \mathrm{H}-3,144-146$ & 236.45 & 264.81 & 4.35 & 25.66 & 0.030 & 0.80 & 8.1 \\
\hline $26 \mathrm{H}-4,4-6$ & 236.55 & 264.91 & 7.62 & 17.99 & 0.053 & 1.41 & 14.0 \\
\hline $26 \mathrm{H}-4,14-16$ & 236.65 & 265.01 & 8.26 & 14.15 & 0.049 & 1.36 & 15.6 \\
\hline $26 \mathrm{H}-4,24-26$ & 236.75 & 265.11 & 5.12 & 22.50 & 0.031 & 0.89 & 9.0 \\
\hline $26 \mathrm{H}-4,34-36$ & 236.85 & 265.21 & 4.31 & 20.67 & 0.033 & 0.66 & 8.0 \\
\hline $26 \mathrm{H}-4,44-46$ & 236.95 & 265.31 & 3.62 & 19.52 & 0.038 & 0.45 & 6.7 \\
\hline $26 \mathrm{H}-4,54-56$ & 237.05 & 265.41 & 4.84 & 19.22 & 0.033 & 0.77 & 8.9 \\
\hline $26 \mathrm{H}-4,64-66$ & 237.15 & 265.51 & 8.29 & 10.89 & 0.049 & 0.95 & 16.2 \\
\hline $26 \mathrm{H}-4,74-76$ & 237.25 & 265.61 & 6.12 & 15.80 & 0.037 & 0.69 & 11.3 \\
\hline $26 \mathrm{H}-4,84-86$ & 237.35 & 265.71 & 3.92 & 20.46 & 0.038 & 0.64 & 7.4 \\
\hline $26 \mathrm{H}-4,96-98$ & 237.47 & 265.83 & 5.64 & 16.94 & 0.045 & 0.64 & 10.6 \\
\hline $26 \mathrm{H}-4,108-110$ & 237.59 & 265.95 & 6.32 & 16.80 & 0.028 & 0.88 & 11.6 \\
\hline $26 \mathrm{H}-4,115-117$ & 237.66 & 266.02 & 6.54 & 14.58 & 0.041 & 0.72 & 12.2 \\
\hline $26 \mathrm{H}-4,124-126$ & 237.75 & 266.11 & 10.27 & 10.12 & 0.065 & 1.51 & 19.2 \\
\hline $26 \mathrm{H}-4,134-136$ & 237.85 & 266.21 & 6.38 & 16.23 & 0.040 & 1.05 & 12.3 \\
\hline $26 \mathrm{H}-4,144-146$ & 237.95 & 266.31 & 2.99 & 28.01 & 0.030 & 0.67 & 5.7 \\
\hline 154-926B- & & & & & & & \\
\hline $50 X-1,14-16$ & 461.55 & 491.36 & 4.48 & 14.73 & 0.049 & 0.69 & 10.2 \\
\hline $50 X-1,24-26$ & 461.65 & 491.46 & 4.57 & 14.50 & 0.043 & 0.70 & 10.5 \\
\hline $50 X-1,34-36$ & 461.75 & 491.56 & 3.89 & 15.37 & 0.046 & 0.61 & 9.1 \\
\hline $50 X-1,44-46$ & 461.85 & 491.66 & 3.93 & 15.23 & 0.039 & 0.64 & 9.2 \\
\hline $50 X-1,52-54$ & 461.93 & 491.74 & 3.26 & 15.87 & 0.043 & 0.61 & 7.8 \\
\hline $50 X-1,64-66$ & 462.05 & 491.86 & 3.40 & 16.28 & 0.045 & 0.49 & 7.4 \\
\hline $50 X-1,74-76$ & 462.15 & 491.96 & 2.98 & 16.17 & 0.032 & 0.50 & 7.5 \\
\hline $50 X-1,85-87$ & 462.26 & 492.07 & 3.30 & 16.71 & 0.036 & 0.43 & 8.0 \\
\hline $50 X-1,94-96$ & 462.35 & 492.16 & 3.07 & 16.09 & 0.039 & 0.53 & 7.2 \\
\hline $50 X-1,105-107$ & 462.45 & 492.26 & 3.82 & 15.62 & 0.047 & 0.52 & 8.7 \\
\hline $50 X-1,114-116$ & 462.55 & 492.36 & 4.54 & 17.54 & 0.069 & 0.59 & 10.7 \\
\hline $50 X-1,124-126$ & 462.65 & 492.46 & 5.23 & 14.65 & 0.060 & 0.62 & 11.6 \\
\hline $50 X-1,134-136$ & 462.75 & 492.56 & 5.61 & 14.15 & 0.047 & 0.73 & 12.5 \\
\hline $50 X-1,144-146$ & 462.85 & 492.66 & 5.35 & 14.45 & 0.050 & 0.73 & 12.1 \\
\hline $50 X-2,4-6$ & 462.95 & 492.76 & 3.68 & 13.35 & 0.042 & 0.52 & 8.8 \\
\hline $50 X-2,14-16$ & 463.05 & 492.86 & 3.50 & 16.73 & 0.037 & 0.54 & 8.6 \\
\hline $50 X-2,24-26$ & 463.15 & 492.96 & 3.35 & 18.58 & 0.026 & 0.55 & 8.2 \\
\hline $50 X-2,34-36$ & 463.25 & 493.06 & 4.35 & 12.88 & 0.027 & 0.51 & 9.9 \\
\hline $50 X-2,44-46$ & 463.35 & 493.16 & 4.39 & 17.13 & 0.027 & 0.63 & 10.6 \\
\hline $50 X-2,54-56$ & 463.45 & 493.26 & 4.89 & 17.65 & 0.028 & 0.66 & 11.4 \\
\hline $50 X-2,64-66$ & 463.55 & 493.36 & 5.06 & 12.22 & 0.036 & 0.59 & 11.0 \\
\hline $50 X-2,74-76$ & 463.65 & 493.46 & 4.82 & 12.74 & 0.047 & 0.55 & 10.3 \\
\hline $50 X-2,84-86$ & 463.75 & 493.56 & 6.71 & 13.32 & 0.043 & 0.77 & 14.6 \\
\hline $50 X-2,94-96$ & 463.85 & 493.66 & 5.41 & 14.70 & 0.037 & 0.69 & 12.5 \\
\hline $50 X-2,104-106$ & 463.95 & 493.76 & 3.10 & 13.52 & 0.033 & 0.56 & 7.5 \\
\hline $50 X-2,114-116$ & 464.05 & 493.86 & 3.03 & 19.51 & 0.040 & 0.54 & 7.4 \\
\hline $50 X-2,124-126$ & 464.15 & 493.96 & 3.91 & 16.45 & 0.047 & 0.57 & 9.3 \\
\hline $50 X-2,134-136$ & 464.25 & 494.06 & 3.49 & 17.17 & 0.042 & 0.68 & 9.8 \\
\hline $50 X-2,146-148$ & 464.37 & 494.18 & 3.78 & 16.79 & 0.022 & 0.51 & 9.5 \\
\hline $50 X-3,4-6$ & 464.45 & 494.26 & 2.73 & 22.61 & 0.020 & 0.39 & 6.7 \\
\hline $50 X-3,14-16$ & 464.55 & 494.36 & 3.93 & 18.36 & 0.023 & 0.55 & 9.4 \\
\hline $50 X-3,24-26$ & 464.65 & 494.46 & 4.14 & 19.50 & 0.027 & 0.59 & 9.6 \\
\hline $50 X-3,30-32$ & 464.75 & 494.56 & 4.96 & 17.41 & 0.033 & 0.66 & 11.7 \\
\hline $50 \times-3,44-46$ & 464.85 & 494.66 & 3.80 & 18.58 & 0.092 & 0.62 & 9.7 \\
\hline $50 X-3,54-56$ & 464.95 & 494.76 & 3.43 & 19.11 & 0.066 & 0.56 & 9.0 \\
\hline $50 X-3,64-66$ & 465.05 & 494.86 & 2.98 & 22.05 & 0.055 & 0.55 & 7.2 \\
\hline $50 X-3,73-75$ & 465.14 & 494.95 & 2.99 & 18.86 & 0.043 & 0.53 & 7.7 \\
\hline $50 X-3,84-86$ & 465.25 & 495.06 & 3.54 & 18.52 & 0.035 & 0.51 & 9.5 \\
\hline $50 X-3,93-95$ & 465.35 & 495.16 & 3.93 & 18.43 & 0.043 & 0.66 & 9.7 \\
\hline $50 X-3,104-106$ & 465.45 & 495.26 & 3.65 & 20.61 & 0.041 & 0.69 & 8.9 \\
\hline $50 X-3,114-116$ & 465.55 & 495.36 & 5.00 & 17.41 & 0.056 & 0.91 & 12.2 \\
\hline $50 X-3,124-126$ & 465.65 & 495.46 & 5.62 & 16.51 & 0.050 & 0.78 & 13.6 \\
\hline $50 X-3,134-136$ & 465.75 & 495.56 & 6.85 & 15.71 & 0.053 & 0.84 & 16.2 \\
\hline $50 X-3,144-146$ & 465.85 & 495.66 & 5.79 & 16.90 & 0.070 & 0.89 & 13.3 \\
\hline $50 X-4,4-6$ & 465.95 & 495.76 & 3.73 & 19.45 & 0.045 & 0.86 & 9.2 \\
\hline $50 X-4,14-16$ & 466.05 & 495.86 & 5.08 & 22.98 & 0.059 & 0.78 & 12.9 \\
\hline $50 X-4,24-26$ & 466.15 & 495.96 & 4.22 & 18.91 & 0.053 & 0.92 & 10.1 \\
\hline $50 X-4,32-34$ & 466.23 & 496.04 & 4.15 & 23.60 & 0.048 & 0.80 & 10.9 \\
\hline $50 X-4,44-46$ & 466.35 & 496.16 & 5.00 & 22.82 & 0.058 & 0.73 & 12.0 \\
\hline $50 X-4,54-56$ & 466.45 & 496.26 & 4.40 & 18.56 & 0.056 & 0.68 & 10.2 \\
\hline $50 X-4,64-66$ & 466.55 & 496.36 & 7.23 & 20.34 & 0.051 & 0.89 & 16.3 \\
\hline $50 X-4,74-76$ & 466.65 & 496.46 & 7.34 & 16.68 & 0.052 & 1.01 & 16.5 \\
\hline $50 X-4,84-86$ & 466.75 & 496.56 & 5.58 & 18.87 & 0.084 & 0.74 & 12.8 \\
\hline $50 X-4,96-98$ & 466.85 & 496.66 & 4.90 & 22.44 & 0.069 & 0.72 & 12.2 \\
\hline 50X-4, 104-106 & 466.95 & 496.76 & 5.03 & 21.07 & 0.051 & 0.66 & 10.8 \\
\hline $50 X-4,114-116$ & 467.05 & 496.86 & 4.04 & 19.01 & 0.047 & 0.63 & 9.9 \\
\hline
\end{tabular}


Table 3 (continued).

\begin{tabular}{|c|c|c|c|c|c|c|}
\hline $\begin{array}{l}\text { Core, section, } \\
\text { interval }(\mathrm{cm})\end{array}$ & $\begin{array}{l}\text { Depth } \\
\text { (mbsf) }\end{array}$ & $\begin{array}{l}\text { Depth } \\
\text { (mcd) }\end{array}$ & $\begin{array}{c}\mathrm{Al} \\
(\%)\end{array}$ & $\begin{array}{l}\mathrm{Ca} \\
(\%)\end{array}$ & $\begin{array}{l}\mathrm{P} \\
(\%)\end{array}$ & $\begin{array}{c}\mathrm{K} \\
(\%)\end{array}$ \\
\hline $50 \mathrm{X}-4,124-126$ & 467.15 & 496.96 & 3.40 & 19.50 & 0.042 & 0.62 \\
\hline $50 \mathrm{X}-4,134-136$ & 467.25 & 497.06 & 4.40 & 20.36 & 0.055 & 0.66 \\
\hline $50 \mathrm{X}-4,144-146$ & 467.35 & 497.16 & 4.99 & 21.25 & 0.042 & 0.70 \\
\hline $50 X-5,4-6$ & 467.45 & 497.26 & 3.44 & 16.31 & 0.038 & 0.54 \\
\hline $50 X-5,14-16$ & 467.55 & 497.36 & 3.81 & 14.34 & 0.048 & 0.52 \\
\hline $50 X-5,24-26$ & 467.65 & 497.46 & 4.52 & 15.05 & 0.040 & 0.56 \\
\hline $50 X-5,32-34$ & 467.73 & 497.54 & 4.86 & 13.36 & 0.037 & 0.66 \\
\hline $50 X-5,44-46$ & 467.85 & 497.66 & 4.29 & 15.21 & 0.061 & 0.47 \\
\hline $50 X-5,52-54$ & 467.93 & 497.74 & 4.43 & 14.96 & 0.039 & 0.60 \\
\hline $50 X-5,64-66$ & 468.05 & 497.86 & 3.32 & 21.04 & 0.045 & 0.50 \\
\hline $50 X-5,74-76$ & 468.15 & 497.96 & 2.83 & 16.66 & 0.037 & 0.42 \\
\hline $50 X-5,84-86$ & 468.25 & 498.06 & 2.66 & 15.09 & 0.029 & 0.45 \\
\hline $50 X-5,96-98$ & 468.37 & 498.18 & 2.99 & 21.65 & 0.024 & 0.42 \\
\hline $50 X-5,104-106$ & 468.45 & 498.26 & 2.78 & 16.76 & 0.032 & 0.38 \\
\hline $50 X-5,114-116$ & 468.55 & 498.36 & 2.94 & 16.40 & 0.038 & 0.39 \\
\hline $50 X-5,124-126$ & 468.65 & 498.46 & 3.56 & 16.29 & 0.037 & 0.47 \\
\hline $50 \mathrm{X}-5,134-136$ & 468.75 & 498.56 & 3.85 & 15.74 & 0.040 & 0.48 \\
\hline $50 X-5,144-146$ & 468.85 & 498.66 & 3.64 & 16.26 & 0.043 & 0.50 \\
\hline $50 X-6,4-6$ & 468.95 & 498.76 & 5.05 & 17.46 & 0.037 & 0.67 \\
\hline $50 X-6,14-16$ & 469.05 & 498.86 & 5.56 & 17.41 & 0.040 & 0.67 \\
\hline $50 X-6,22-24$ & 469.13 & 498.94 & 6.28 & 16.57 & 0.037 & 0.79 \\
\hline $50 X-6,34-36$ & 469.25 & 499.06 & 5.44 & 16.64 & 0.045 & 0.69 \\
\hline $50 X-6,46-48$ & 469.37 & 499.18 & 4.02 & 14.89 & 0.038 & 0.54 \\
\hline $50 X-6,54-56$ & 469.45 & 499.26 & 4.83 & 13.73 & 0.038 & 0.61 \\
\hline $50 X-6,64-66$ & 469.55 & 499.36 & 5.61 & 17.56 & 0.039 & 0.67 \\
\hline $50 X-6,74-76$ & 469.65 & 499.46 & 4.18 & 17.77 & 0.043 & 0.58 \\
\hline $50 X-6,84-86$ & 469.75 & 499.56 & 3.28 & 13.36 & 0.030 & 0.41 \\
\hline $50 X-6,94-96$ & 469.85 & 499.66 & 5.50 & 16.47 & 0.045 & 0.62 \\
\hline $50 \mathrm{X}-6,104-106$ & 469.95 & 499.76 & 5.97 & 16.24 & 0.048 & 0.76 \\
\hline $50 X-6,114-116$ & 470.05 & 499.86 & 4.77 & 14.04 & 0.038 & 0.58 \\
\hline $50 \mathrm{X}-6,124-126$ & 470.15 & 499.96 & 6.04 & 16.11 & 0.047 & 0.74 \\
\hline $50 \mathrm{X}-6,134-136$ & 470.25 & 500.06 & 4.58 & 13.89 & 0.034 & 0.57 \\
\hline $50 \mathrm{X}-\mathrm{CC}, 4-6$ & 470.35 & 500.16 & 3.58 & 16.73 & 0.056 & 0.55 \\
\hline 50X-CC, $14-16$ & 470.45 & 500.26 & 3.57 & 17.23 & 0.045 & 0.52 \\
\hline $50 \mathrm{X}-\mathrm{CC}, 24-26$ & 470.55 & 500.36 & 3.75 & 16.77 & 0.047 & 0.50 \\
\hline $50 \mathrm{X}-\mathrm{CC}, 34-36$ & 470.65 & 500.46 & 4.15 & 15.76 & 0.044 & 0.59 \\
\hline $50 \mathrm{X}-\mathrm{CC}, 44-46$ & 470.75 & 500.56 & 4.44 & 15.24 & 0.049 & 0.55 \\
\hline $51 X-1,14-16$ & 471.15 & 500.96 & 4.70 & 16.76 & 0.034 & 0.48 \\
\hline $51 X-1,24-26$ & 471.25 & 501.06 & 4.90 & 16.04 & 0.028 & 0.52 \\
\hline $51 \mathrm{X}-1,33-35$ & 471.34 & 501.15 & 5.75 & 21.66 & 0.045 & 0.83 \\
\hline $51 X-1,44-46$ & 471.45 & 501.26 & 3.98 & 24.29 & 0.050 & 0.68 \\
\hline $51 X-1,54-56$ & 471.55 & 501.36 & 2.96 & 18.27 & 0.043 & 0.49 \\
\hline $51 X-1,64-66$ & 471.65 & 501.46 & 3.10 & 17.88 & 0.050 & 0.46 \\
\hline $51 X-1,74-76$ & 471.75 & 501.56 & 3.64 & 16.83 & 0.037 & 0.41 \\
\hline $51 X-1,84-86$ & 471.85 & 501.66 & 4.40 & 16.23 & 0.034 & 0.56 \\
\hline $51 X-1,93-95$ & 471.94 & 501.75 & 5.26 & 18.38 & 0.045 & 0.66 \\
\hline $51 \mathrm{X}-1,104-106$ & 472.05 & 501.86 & 5.64 & 17.55 & 0.052 & 0.66 \\
\hline $51 \mathrm{X}-1,114-116$ & 472.15 & 501.96 & 5.56 & 18.13 & 0.044 & 0.66 \\
\hline $51 X-1,124-126$ & 472.25 & 502.06 & 5.69 & 17.67 & 0.032 & 0.71 \\
\hline $51 \mathrm{X}-1,134-136$ & 472.35 & 502.16 & 6.01 & 17.43 & 0.032 & 0.71 \\
\hline $51 \mathrm{X}-1,144-146$ & 472.45 & 502.26 & 4.52 & 15.96 & 0.039 & 0.47 \\
\hline $51 X-2,6-8$ & 472.57 & 502.38 & 4.59 & 18.92 & 0.026 & 0.60 \\
\hline $51 X-2,14-16$ & 472.65 & 502.46 & 3.51 & 19.24 & 0.037 & 0.59 \\
\hline $51 X-2,24-26$ & 472.75 & 502.56 & 3.33 & 20.56 & 0.035 & 0.78 \\
\hline $51 X-2,33-35$ & 472.84 & 502.65 & 3.98 & 19.41 & 0.036 & 0.95 \\
\hline $51 X-2,44-46$ & 472.95 & 502.76 & 3.70 & 20.04 & 0.036 & 0.86 \\
\hline $51 X-2,54-56$ & 473.05 & 502.86 & 4.27 & 20.35 & 0.031 & 0.60 \\
\hline $51 X-2,64-66$ & 473.15 & 502.96 & 5.45 & 17.21 & 0.037 & 0.71 \\
\hline $51 X-2,74-76$ & 473.25 & 503.06 & 5.92 & 17.18 & 0.043 & 0.77 \\
\hline $51 X-2,84-86$ & 473.35 & 503.16 & 4.36 & 19.48 & 0.044 & 0.78 \\
\hline $51 X-2,94-96$ & 473.45 & 503.26 & 4.39 & 19.47 & 0.045 & 0.63 \\
\hline $51 X-2,104-106$ & 473.55 & 503.36 & 3.05 & 21.90 & 0.041 & 0.68 \\
\hline $51 X-2,114-116$ & 473.65 & 503.46 & 3.28 & 21.30 & 0.032 & 0.56 \\
\hline $51 \mathrm{X}-2,124-126$ & 473.75 & 503.56 & 3.81 & 20.26 & 0.036 & 0.73 \\
\hline $51 X-2,134-136$ & 473.85 & 503.66 & 2.82 & 22.02 & 0.034 & 0.60 \\
\hline $51 X-2,144-146$ & 473.95 & 503.76 & 2.95 & 21.61 & 0.028 & 0.44 \\
\hline $51 \mathrm{X}-3,3-5$ & 474.04 & 503.85 & 3.18 & 22.78 & 0.035 & 0.55 \\
\hline $51 X-3,14-16$ & 474.15 & 503.96 & 3.33 & 23.28 & 0.031 & 0.70 \\
\hline $51 X-3,24-26$ & 474.25 & 504.06 & 5.09 & 19.36 & 0.027 & 0.63 \\
\hline $51 X-3,33-35$ & 474.34 & 504.15 & 6.27 & 15.32 & 0.030 & 0.80 \\
\hline $51 X-3,44-46$ & 474.45 & 504.26 & 4.67 & 20.25 & 0.063 & 0.63 \\
\hline $51 X-3,54-56$ & 474.55 & 504.36 & 4.44 & 20.52 & 0.037 & 0.50 \\
\hline $51 X-3,64-66$ & 474.65 & 504.46 & 3.61 & 21.99 & 0.035 & 0.67 \\
\hline $51 X-3,74-76$ & 474.75 & 504.56 & 3.13 & 21.72 & 0.036 & 0.64 \\
\hline $51 X-3,82-84$ & 474.85 & 504.66 & 3.92 & 20.50 & 0.035 & 0.55 \\
\hline $51 X-3,94-96$ & 474.95 & 504.76 & 3.98 & 21.89 & 0.035 & 0.37 \\
\hline $51 \mathrm{X}-3,104-106$ & 475.05 & 504.86 & 3.11 & 22.91 & 0.030 & 0.53 \\
\hline $51 X-3,114-116$ & 475.15 & 504.96 & 3.31 & 21.97 & 0.035 & 0.40 \\
\hline $51 X-3,124-126$ & 475.25 & 505.06 & 3.95 & 21.50 & 0.029 & 0.45 \\
\hline $51 X-3,134-136$ & 475.35 & 505.16 & 3.95 & 20.32 & 0.026 & 0.68 \\
\hline $51 X-4,4-6$ & 475.55 & 505.36 & 4.75 & 20.97 & 0.043 & 0.63 \\
\hline $51 X-4,14-16$ & 475.65 & 505.46 & 5.00 & 18.52 & 0.038 & 0.69 \\
\hline $51 X-4,24-26$ & 475.75 & 505.56 & 4.24 & 22.12 & 0.048 & 0.65 \\
\hline $51 X-4,33-35$ & 475.84 & 505.65 & 3.65 & 22.86 & 0.040 & 0.48 \\
\hline $51 X-4,47-49$ & 475.98 & 505.79 & 3.32 & 23.75 & 0.040 & 0.55 \\
\hline $51 X-4,54-56$ & 476.05 & 505.86 & 3.30 & 23.57 & 0.029 & 0.50 \\
\hline $51 X-4,64-66$ & 476.15 & 505.96 & 2.90 & 22.51 & 0.028 & 0.46 \\
\hline $51 X-4,74-76$ & 476.25 & 506.06 & 4.18 & 22.10 & 0.026 & 0.61 \\
\hline $51 X-4,84-86$ & 476.35 & 506.16 & 4.43 & 21.45 & 0.030 & 0.66 \\
\hline $51 X-4,93-95$ & 476.44 & 506.25 & 3.97 & 22.39 & 0.034 & 0.61 \\
\hline $51 X-4,104-106$ & 476.55 & 506.36 & 3.94 & 22.56 & 0.026 & 0.65 \\
\hline
\end{tabular}


Table 3 (continued).

\begin{tabular}{|c|c|c|c|c|c|c|}
\hline $\begin{array}{l}\text { Core, section, } \\
\text { interval }(\mathrm{cm})\end{array}$ & $\begin{array}{l}\text { Depth } \\
\text { (mbsf) }\end{array}$ & $\begin{array}{l}\text { Depth } \\
\text { (mcd) }\end{array}$ & $\begin{array}{l}\mathrm{Al} \\
(\%)\end{array}$ & $\begin{array}{c}\mathrm{Ca} \\
(\%)\end{array}$ & $\begin{array}{c}\mathrm{P} \\
(\%)\end{array}$ & $\begin{array}{c}\mathrm{K} \\
(\%)\end{array}$ \\
\hline $51 X-4,114-116$ & 476.65 & 506.46 & 3.10 & 21.82 & 0.032 & 0.52 \\
\hline $51 \mathrm{X}-4,124-126$ & 476.75 & 506.56 & 4.24 & 20.01 & 0.032 & 0.61 \\
\hline $51 \mathrm{X}-4,136-138$ & 476.87 & 506.68 & 3.82 & 19.98 & 0.033 & 0.64 \\
\hline $51 X-4,144-146$ & 476.95 & 506.76 & 2.83 & 22.32 & 0.037 & 0.60 \\
\hline $51 X-5,4-6$ & 477.05 & 506.86 & 3.40 & 26.28 & 0.039 & 0.70 \\
\hline $51 X-5,14-16$ & 477.15 & 506.96 & 3.72 & 18.95 & 0.023 & 0.56 \\
\hline $51 X-5,24-26$ & 477.25 & 507.06 & 5.24 & 15.03 & 0.033 & 0.82 \\
\hline $51 X-5,33-35$ & 477.34 & 507.15 & 6.76 & 20.37 & 0.037 & 1.02 \\
\hline $51 X-5,44-46$ & 477.45 & 507.26 & 4.67 & 17.18 & 0.050 & 0.76 \\
\hline $51 X-5,52-54$ & 477.55 & 507.36 & 3.93 & 18.90 & 0.038 & 0.58 \\
\hline $51 X-5,64-66$ & 477.65 & 507.46 & 4.94 & 23.70 & 0.059 & 0.77 \\
\hline $51 X-5,74-76$ & 477.75 & 507.56 & 5.18 & 22.84 & 0.048 & 0.82 \\
\hline $51 X-5,84-86$ & 477.85 & 507.66 & 3.79 & 18.63 & 0.031 & 0.56 \\
\hline $51 X-5,93-95$ & 477.94 & 507.75 & 3.28 & 20.00 & 0.035 & 0.46 \\
\hline $51 X-5,104-106$ & 478.05 & 507.86 & 4.09 & 24.58 & 0.045 & 0.60 \\
\hline $51 X-5,114-116$ & 478.15 & 507.96 & 3.61 & 19.20 & 0.030 & 0.50 \\
\hline $51 X-5,124-126$ & 478.25 & 508.06 & 4.07 & 18.84 & 0.033 & 0.58 \\
\hline $51 X-5,134-136$ & 478.35 & 508.16 & 4.86 & 17.50 & 0.039 & 0.74 \\
\hline $51 X-5,144-145$ & 478.45 & 508.26 & 5.14 & 16.82 & 0.034 & 0.80 \\
\hline $51 X-6,4-6$ & 478.55 & 508.36 & 4.40 & 18.75 & 0.046 & 0.66 \\
\hline $51 X-6,14-16$ & 478.65 & 508.46 & 3.85 & 16.26 & 0.036 & 0.61 \\
\hline $51 X-6,24-26$ & 478.75 & 508.56 & 3.65 & 16.57 & 0.043 & 0.60 \\
\hline $51 X-6,33-35$ & 478.84 & 508.65 & 3.41 & 16.67 & 0.040 & 0.67 \\
\hline $51 X-6,44-46$ & 478.95 & 508.76 & 5.05 & 22.68 & 0.053 & 0.89 \\
\hline $51 X-6,54-56$ & 479.05 & 508.86 & 5.38 & 21.87 & 0.043 & 0.93 \\
\hline $51 X-6,64-66$ & 479.15 & 508.96 & 4.46 & 15.50 & 0.033 & 0.72 \\
\hline $51 X-6,74-76$ & 479.25 & 509.06 & 5.22 & 22.45 & 0.044 & 0.74 \\
\hline $51 X-6,84-86$ & 479.35 & 509.16 & 4.22 & 15.31 & 0.032 & 0.60 \\
\hline $51 X-6,94-96$ & 479.45 & 509.26 & 4.50 & 15.37 & 0.031 & 0.69 \\
\hline $51 X-6,104-106$ & 479.55 & 509.36 & 3.88 & 19.27 & 0.036 & 0.63 \\
\hline $51 X-6,114-116$ & 479.65 & 509.46 & 2.87 & 16.83 & 0.034 & 0.52 \\
\hline $51 \mathrm{X}-6,124-126$ & 479.75 & 509.56 & 3.01 & 20.37 & 0.037 & 0.57 \\
\hline $51 \mathrm{X}-6,134-136$ & 479.85 & 509.66 & 2.98 & 20.22 & 0.041 & 0.52 \\
\hline $51 X-6,144-146$ & 479.95 & 509.76 & 3.38 & 19.68 & 0.036 & 0.53 \\
\hline $51 X-7,4-6$ & 480.05 & 509.86 & 4.42 & 17.02 & 0.043 & 0.65 \\
\hline $51 X-7,14-16$ & 480.15 & 509.96 & 4.36 & 15.92 & 0.045 & 0.63 \\
\hline $51 X-7,24-26$ & 480.25 & 510.06 & 5.44 & 15.47 & 0.040 & 0.71 \\
\hline $51 X-7,33-35$ & 480.34 & 510.15 & 5.06 & 16.15 & 0.051 & 0.69 \\
\hline 51X-CC, 4-6 & 480.45 & 510.26 & 3.55 & 17.21 & 0.038 & 0.56 \\
\hline $51 \mathrm{X}-\mathrm{CC}, 14-16$ & 480.55 & 510.36 & 3.38 & 17.60 & 0.036 & 0.52 \\
\hline $51 \mathrm{X}-\mathrm{CC}, 24-26$ & 480.65 & 510.46 & 3.21 & 17.73 & 0.033 & 0.53 \\
\hline $51 \mathrm{X}-\mathrm{CC}, 34-36$ & 480.75 & 510.56 & 3.21 & 17.59 & 0.035 & 0.59 \\
\hline $64 X-1,4-6$ & 596.55 & 626.36 & 3.36 & 22.16 & 0.038 & 0.53 \\
\hline $64 X-1,14-15$ & 596.65 & 626.46 & 2.83 & 22.80 & 0.043 & 0.49 \\
\hline $64 X-1,24-26$ & 596.75 & 626.56 & 2.51 & 22.89 & 0.035 & 0.38 \\
\hline $64 X-1,35-36$ & 596.86 & 626.67 & 1.88 & 24.16 & 0.031 & 0.31 \\
\hline $64 X-1,44-46$ & 596.95 & 626.76 & 1.86 & 23.55 & 0.026 & 0.27 \\
\hline $64 X-1,54-55$ & 597.05 & 626.86 & 2.09 & 18.91 & 0.034 & 0.32 \\
\hline $64 X-1,64-66$ & 597.15 & 626.96 & 1.97 & 19.11 & 0.034 & 0.33 \\
\hline $64 X-1,74-75$ & 597.25 & 627.06 & 2.38 & 22.43 & 0.024 & 0.38 \\
\hline $64 X-1,84-86$ & 597.35 & 627.16 & 3.36 & 22.23 & 0.035 & 0.56 \\
\hline $64 \mathrm{X}-1,94-95$ & 597.45 & 627.26 & 2.96 & 17.84 & 0.040 & 0.46 \\
\hline $64 \mathrm{X}-1,104-106$ & 597.55 & 627.36 & 3.00 & 17.46 & 0.032 & 0.48 \\
\hline $64 X-1,114-115$ & 597.65 & 627.46 & 2.56 & 23.56 & 0.029 & 0.43 \\
\hline $64 X-1,124-126$ & 597.75 & 627.56 & 2.30 & 23.90 & 0.037 & 0.42 \\
\hline $64 \mathrm{X}-1,134-135$ & 597.85 & 627.66 & 2.14 & 18.52 & 0.033 & 0.36 \\
\hline $64 \mathrm{X}-1,143-145$ & 597.94 & 627.75 & 2.20 & 23.26 & 0.030 & 0.42 \\
\hline $64 X-2,4-5$ & 598.05 & 627.86 & 2.10 & 18.36 & 0.039 & 0.37 \\
\hline $64 X-2,14-16$ & 598.15 & 627.96 & 1.66 & 18.99 & 0.046 & 0.36 \\
\hline $64 X-2,24-25$ & 598.25 & 628.06 & 1.93 & 18.85 & 0.038 & 0.35 \\
\hline $64 X-2,34-36$ & 598.35 & 628.16 & 2.60 & 18.37 & 0.033 & 0.43 \\
\hline $64 X-2,44-45$ & 598.45 & 628.26 & 3.17 & 17.75 & 0.038 & 0.54 \\
\hline $64 X-2,54-56$ & 598.55 & 628.36 & 3.37 & 17.60 & 0.035 & 0.57 \\
\hline $64 X-2,64-65$ & 598.65 & 628.46 & 4.09 & 16.33 & 0.040 & 0.66 \\
\hline $64 X-2,74-76$ & 598.75 & 628.56 & 4.92 & 15.90 & 0.038 & 0.76 \\
\hline $64 X-2,84-86$ & 598.85 & 628.66 & 4.12 & 16.22 & 0.028 & 0.69 \\
\hline $64 X-2,94-96$ & 598.95 & 628.76 & 3.45 & 17.62 & 0.036 & 0.59 \\
\hline $64 \mathrm{X}-2,104-105$ & 599.05 & 628.86 & 2.82 & 19.94 & 0.033 & 0.49 \\
\hline $64 X-2,114-116$ & 599.15 & 628.96 & 2.28 & 19.03 & 0.028 & 0.33 \\
\hline $64 \mathrm{X}-2,124-125$ & 599.25 & 629.06 & 2.25 & 19.22 & 0.034 & 0.42 \\
\hline $64 X-2,134-136$ & 599.35 & 629.16 & 2.19 & 19.22 & 0.031 & 0.39 \\
\hline $64 X-2,146-147$ & 599.45 & 629.26 & 2.10 & 19.41 & 0.030 & 0.39 \\
\hline $64 X-3,4-6$ & 599.55 & 629.36 & 3.00 & 21.72 & 0.043 & 0.43 \\
\hline $64 X-3,14-16$ & 599.65 & 629.46 & 3.47 & 20.58 & 0.048 & 0.45 \\
\hline $64 X-3,24-26$ & 599.75 & 629.56 & 3.32 & 20.62 & 0.044 & 0.52 \\
\hline $64 X-3,33-34$ & 599.84 & 629.65 & 3.43 & 20.77 & 0.045 & 0.48 \\
\hline $64 X-3,43-45$ & 599.94 & 629.75 & 3.98 & 21.29 & 0.038 & 0.52 \\
\hline $64 X-3,54-56$ & 600.05 & 629.86 & 8.76 & 15.06 & 0.029 & 0.64 \\
\hline $64 X-3,67-69$ & 600.18 & 629.99 & 4.04 & 20.70 & 0.059 & 0.65 \\
\hline $64 X-3,74-76$ & 600.25 & 630.06 & 2.39 & 22.56 & 0.048 & 0.44 \\
\hline $64 X-3,84-86$ & 600.35 & 630.16 & 1.69 & 21.74 & 0.042 & 0.37 \\
\hline $64 X-3,94-96$ & 600.45 & 630.26 & 1.68 & 22.02 & 0.038 & 0.35 \\
\hline $64 \mathrm{X}-3,105-107$ & 600.56 & 630.37 & 1.75 & 18.62 & 0.026 & 0.32 \\
\hline $64 X-3,114-116$ & 600.65 & 630.46 & 3.11 & 19.76 & 0.038 & 0.52 \\
\hline $64 X-3,124-126$ & 600.75 & 630.56 & 4.19 & 19.30 & 0.048 & 0.68 \\
\hline $64 X-3,134-136$ & 600.85 & 630.66 & 4.06 & 19.00 & 0.046 & 0.65 \\
\hline $64 X-3,144-146$ & 600.95 & 630.76 & 4.57 & 18.55 & 0.050 & 0.68 \\
\hline $64 X-4,4-5$ & 601.05 & 630.86 & 3.45 & 18.53 & 0.036 & 0.57 \\
\hline $64 X-4,14-16$ & 601.15 & 630.96 & 2.89 & 13.97 & 0.031 & 0.50 \\
\hline $64 X-4,24-26$ & 601.25 & 631.06 & 3.07 & 18.85 & 0.041 & 0.51 \\
\hline & 601.33 & 631.14 & 2.15 & 20.37 & 0.034 & 0.40 \\
\hline
\end{tabular}


Table 3 (continued).

\begin{tabular}{|c|c|c|c|c|c|c|c|}
\hline $\begin{array}{l}\text { Core, section, } \\
\text { interval }(\mathrm{cm})\end{array}$ & $\begin{array}{l}\text { Depth } \\
\text { (mbsf) }\end{array}$ & $\begin{array}{l}\text { Depth } \\
\text { (mcd) }\end{array}$ & $\begin{array}{c}\mathrm{Al} \\
(\%)\end{array}$ & $\begin{array}{l}\mathrm{Ca} \\
(\%)\end{array}$ & $\begin{array}{c}\mathrm{P} \\
(\%)\end{array}$ & $\begin{array}{c}\mathrm{K} \\
(\%)\end{array}$ & $\begin{array}{l}\mathrm{Si} \\
(\%)\end{array}$ \\
\hline $64 X-4,44-46$ & 601.45 & 631.26 & 1.85 & 16.23 & 0.036 & 0.43 & 4.8 \\
\hline $64 \mathrm{X}-4,54-56$ & 601.55 & 631.36 & 2.43 & 19.25 & 0.038 & 0.36 & 6.0 \\
\hline $64 \mathrm{X}-4,64-65$ & 601.65 & 631.46 & 2.83 & 18.70 & 0.036 & 0.49 & 6.8 \\
\hline $64 X-4,72-74$ & 601.73 & 631.54 & 3.48 & 18.95 & 0.029 & 0.59 & 8.1 \\
\hline $64 \mathrm{X}-4,84-85$ & 601.85 & 631.66 & 4.37 & 16.50 & 0.046 & 0.69 & 10.2 \\
\hline $64 X-4,94-96$ & 601.95 & 631.76 & 4.70 & 13.03 & 0.033 & 0.73 & 11.2 \\
\hline $64 \mathrm{X}-4,104-105$ & 602.05 & 631.86 & 3.98 & 18.17 & 0.029 & 0.62 & 10.0 \\
\hline $64 X-4,114-116$ & 602.15 & 631.96 & 1.93 & 13.56 & 0.032 & 0.42 & 4.7 \\
\hline $64 \mathrm{X}-4,124-126$ & 602.25 & 632.06 & 1.69 & 19.58 & 0.041 & 0.33 & 4.4 \\
\hline $64 X-4,134-136$ & 602.35 & 632.16 & 1.57 & 19.65 & 0.043 & 0.30 & 4.3 \\
\hline $64 \mathrm{X}-4,143-144$ & 602.44 & 632.25 & 1.44 & 21.82 & 0.030 & 0.31 & 3.9 \\
\hline $64 X-5,4-6$ & 602.55 & 632.36 & 1.60 & 19.16 & 0.032 & 0.27 & 4.2 \\
\hline $64 \mathrm{X}-5,14-15$ & 602.65 & 632.46 & $\begin{array}{l}1.00 \\
1.87\end{array}$ & 19.11 & 0.026 & 0.33 & 4.7 \\
\hline $64 X-5,24-26$ & 602.75 & 632.56 & 2.26 & 18.35 & 0.028 & 0.34 & 5.4 \\
\hline $64 \mathrm{X}-5,34-35$ & 602.85 & 632.66 & 2.38 & 18.35 & 0.031 & 0.31 & 5.6 \\
\hline $64 X-5,44-46$ & 602.95 & 632.76 & 4.58 & 15.46 & 0.038 & 0.57 & 10.0 \\
\hline $64 X-5,54-55$ & 603.05 & 632.86 & 8.76 & 15.06 & 0.029 & 0.64 & 16.1 \\
\hline $64 X-5,64-66$ & 603.15 & 632.96 & 5.08 & 13.45 & 0.022 & 0.70 & 10.9 \\
\hline $64 X-5,74-76$ & 603.25 & 633.06 & 1.80 & 18.03 & 0.036 & 0.33 & 4.6 \\
\hline $64 \mathrm{X}-5,84-86$ & 603.35 & 633.16 & 1.23 & 19.19 & 0.035 & 0.09 & 3.9 \\
\hline $64 X-5,94-96$ & 603.45 & 633.26 & 1.52 & 18.87 & 0.029 & 0.14 & 4.8 \\
\hline $64 \mathrm{X}-5,104-106$ & 603.55 & 633.36 & 2.24 & 17.68 & 0.030 & 0.36 & 5.6 \\
\hline $64 \mathrm{X}-5,114-115$ & 603.65 & 633.46 & 2.05 & 17.83 & 0.029 & 0.18 & 6.5 \\
\hline $64 \mathrm{X}-5,124-126$ & 603.75 & 633.56 & 2.99 & 16.89 & 0.031 & 0.48 & 7.1 \\
\hline $64 \mathrm{X}-5,134-136$ & 603.85 & 633.66 & 2.75 & 16.82 & 0.033 & 0.43 & 8.1 \\
\hline $64 X-5,143-145$ & 603.94 & 633.75 & 1.72 & 10.22 & 0.019 & 0.10 & 5.1 \\
\hline $64 X-6,4-5$ & 604.05 & 633.86 & 4.99 & 15.74 & 0.029 & 0.70 & 12.2 \\
\hline $64 X-6,15-17$ & 604.15 & 633.96 & 3.20 & 18.51 & 0.043 & 0.45 & 7.4 \\
\hline $64 \mathrm{X}-6,24-25$ & 604.25 & 634.06 & 2.21 & 19.01 & 0.034 & 0.34 & 5.2 \\
\hline $64 \mathrm{X}-6,36-38$ & 604.37 & 634.18 & 2.22 & 19.65 & 0.040 & 0.31 & 5.2 \\
\hline $64 \mathrm{X}-6,44-45$ & 604.45 & 634.26 & 1.67 & 19.51 & 0.033 & 0.24 & 4.2 \\
\hline $64 \mathrm{X}-6,54-56$ & 604.55 & 634.36 & 1.69 & 20.26 & 0.027 & 0.27 & 4.0 \\
\hline $64 \mathrm{X}-6,64-66$ & 604.65 & 634.46 & 2.22 & 23.53 & 0.035 & 0.45 & 5.6 \\
\hline $64 X-6,74-76$ & 604.75 & 634.56 & 3.25 & 24.41 & 0.037 & 0.54 & 7.9 \\
\hline $64 X-6,84-85$ & 604.85 & 634.66 & 3.33 & 17.98 & 0.027 & 0.52 & 7.8 \\
\hline $64 \mathrm{X}-6,94-96$ & 604.85 & 634.76 & 3.55 & 21.77 & 0.035 & 0.63 & 8.5 \\
\hline $64 X-6,104-105$ & 604.95 & 634.86 & 3.35 & 18.08 & 0.035 & 0.46 & 7.7 \\
\hline $64 X-6,114-116$ & 605.05 & 634.96 & 2.84 & 23.20 & 0.035 & 0.53 & 6.8 \\
\hline 64X-6, 124-126 & 605.15 & 635.06 & 2.41 & 24.74 & 0.035 & 0.45 & 6.0 \\
\hline $64 \mathrm{X}-6,135-137$ & 605.26 & 635.17 & 1.63 & 20.10 & 0.033 & 0.27 & 4.0 \\
\hline $64 \mathrm{X}-6,145-146$ & 605.36 & 635.27 & 2.17 & 19.38 & 0.033 & 0.32 & 4.9 \\
\hline $64 X-7,4-6$ & 605.45 & 635.36 & 2.11 & 25.35 & 0.031 & 0.35 & 5.0 \\
\hline $64 X-7,14-15$ & 605.55 & 635.46 & 3.46 & 23.11 & 0.032 & 0.56 & 7.9 \\
\hline $64 X-7,26-28$ & 605.67 & 635.58 & 5.07 & 17.92 & 0.022 & 0.71 & 10.7 \\
\hline $64 X-7,34-35$ & 605.75 & 635.66 & 5.29 & 17.37 & 0.028 & 0.78 & 12.0 \\
\hline $64 \mathrm{X}-\mathrm{CC}, 1-3$ & 605.82 & 635.73 & 3.42 & 20.90 & 0.041 & 0.50 & 7.6 \\
\hline 64X-CC, $14-15$ & 605.95 & 635.86 & 3.95 & 20.49 & 0.049 & 0.52 & 8.3 \\
\hline 64X-CC, $24-26$ & 606.05 & 635.96 & 2.54 & 22.83 & 0.024 & 0.41 & 5.6 \\
\hline 154-929A- & & & & & & & \\
\hline $47 \mathrm{X}-1,0-2$ & 436.11 & 451.53 & 1.81 & 24.00 & 0.042 & 0.45 & 6.1 \\
\hline $47 \mathrm{X}-1,10-12$ & 436.21 & 451.63 & 2.35 & 21.75 & 0.047 & 0.79 & 6.9 \\
\hline $47 \mathrm{X}-1,20-22$ & 436.31 & 451.73 & 2.55 & 21.82 & 0.033 & 0.72 & 8.0 \\
\hline $47 \mathrm{X}-1,30-32$ & 436.41 & 451.83 & 3.09 & 21.14 & 0.033 & 0.70 & 8.5 \\
\hline $47 \mathrm{X}-1,40-42$ & 436.51 & 451.93 & 3.43 & 20.69 & 0.022 & 0.81 & 9.1 \\
\hline $47 \mathrm{X}-1,50-52$ & 436.61 & 452.03 & 4.17 & 20.97 & 0.010 & 0.76 & 10.7 \\
\hline $47 \mathrm{X}-1,60-62$ & 436.71 & 452.13 & 3.68 & 19.94 & 0.045 & 0.67 & 9.9 \\
\hline $47 \mathrm{X}-1,70-72$ & 436.81 & 452.23 & 2.53 & 22.36 & 0.051 & 0.62 & 7.3 \\
\hline $47 \mathrm{X}-1,80-82$ & 436.91 & 452.33 & 2.38 & 24.54 & 0.012 & 0.60 & 6.9 \\
\hline $47 \mathrm{X}-1,90-92$ & 437.01 & 452.43 & 2.34 & 22.63 & 0.050 & 0.51 & 7.2 \\
\hline $47 \mathrm{X}-1,100-102$ & 437.11 & 452.53 & 2.88 & 21.93 & 0.040 & 0.87 & 7.9 \\
\hline $47 \mathrm{X}-1,110-112$ & 437.21 & 452.63 & 3.30 & 22.80 & 0.013 & 0.61 & 8.7 \\
\hline $47 \mathrm{X}-1,120-122$ & 437.31 & 452.73 & 3.58 & 22.35 & 0.015 & 0.60 & 9.6 \\
\hline $47 \mathrm{X}-1,130-132$ & 437.41 & 452.83 & 4.13 & 19.50 & 0.041 & 0.73 & 10.5 \\
\hline $47 \mathrm{X}-1,140-142$ & 437.51 & 452.93 & 3.87 & 21.67 & 0.014 & 0.77 & 9.7 \\
\hline $47 \mathrm{X}-2,0-2$ & 437.61 & 453.03 & 3.44 & 22.83 & 0.039 & 0.41 & 9.1 \\
\hline $47 \mathrm{X}-2,10-12$ & 437.71 & 453.13 & 2.85 & 22.42 & 0.039 & $\begin{array}{l}0.41 \\
0.31\end{array}$ & 7.5 \\
\hline $47 \mathrm{X}-2,20-22$ & 437.81 & 453.23 & 2.62 & 22.23 & 0.044 & 0.29 & 7.2 \\
\hline $47 \mathrm{X}-2,30-32$ & 437.91 & 453.33 & 3.49 & 22.53 & 0.048 & 0.33 & 9.2 \\
\hline $47 \mathrm{X}-2,40-42$ & 438.01 & 453.43 & 3.29 & 22.61 & 0.044 & 0.47 & 9.2 \\
\hline $47 \mathrm{X}-2,50-52$ & 438.11 & 453.53 & 3.36 & 21.27 & 0.033 & 0.48 & 9.1 \\
\hline $47 \mathrm{X}-2,60-62$ & 438.21 & 453.63 & 5.38 & 14.16 & 0.028 & 0.86 & 14.3 \\
\hline $47 \mathrm{X}-2,70-72$ & 438.31 & 453.73 & 5.23 & 15.43 & $\begin{array}{l}0.020 \\
0.020\end{array}$ & 0.77 & 12.9 \\
\hline $47 \mathrm{X}-2,80-82$ & 438.41 & 453.83 & 3.54 & 21.57 & 0.031 & 0.40 & 9.0 \\
\hline $47 X-2,90-92$ & 438.51 & 453.9 & 3.05 & 23.49 & 0.051 & 0.32 & 8.4 \\
\hline $47 \mathrm{X}-2,100-102$ & 438.61 & 454.03 & 2.34 & 22.83 & 0.047 & 0.27 & $\begin{array}{l}0.4 \\
7.2\end{array}$ \\
\hline $47 \mathrm{X}-2,110-112$ & 438.71 & 454.13 & 1.89 & 24.90 & 0.046 & 0.21 & 6.7 \\
\hline $47 \mathrm{X}-2,120-122$ & 438.81 & 454.23 & 1.56 & 20.85 & 0.032 & 0.25 & 7.2 \\
\hline $47 \mathrm{X}-2,130-132$ & 438.91 & 454.33 & 1.65 & 21.07 & 0.022 & 0.20 & 6.1 \\
\hline $47 \mathrm{X}-2,140-142$ & 439.01 & 454.43 & 2.68 & 19.44 & 0.033 & 0.38 & 8.0 \\
\hline $47 \mathrm{X}-3,10-12$ & 439.21 & 454. & 5.66 & 14.88 & 0.0 & 0.86 & $\begin{aligned} 0.0 \\
14.2\end{aligned}$ \\
\hline $47 \mathrm{X}-3,20-22$ & 439.31 & 454.73 & 2.51 & 20.56 & 0.064 & 0.42 & 8.3 \\
\hline $47 \mathrm{X}-3,30-32$ & 439.41 & 454.83 & 4.65 & 17.76 & 0.035 & 0.58 & 11.3 \\
\hline $47 \mathrm{X}-3,40-42$ & 439.51 & 454.93 & 3.56 & 19.72 & 0.035 & 0.52 & 9.2 \\
\hline $47 \mathrm{X}-3,50-52$ & 439.61 & 455.03 & 2.83 & 20.64 & 0.036 & 0.35 & 7.8 \\
\hline $47 X-3,61-63$ & 439.72 & 455.14 & 2.71 & 20.98 & 0.029 & 0.24 & 8.1 \\
\hline$-3,70-72$ & 439.81 & 455.23 & 4.70 & 19.58 & 0.034 & 0.68 & 12.4 \\
\hline $47 \mathrm{X}-3,80-82$ & 439.91 & 455.33 & 4.86 & 17.33 & 0.032 & 0.64 & 12.4 \\
\hline $47 \mathrm{X}-3,90-92$ & 440.01 & 455.43 & 3.14 & 20.08 & 0.026 & 0.46 & 8.7 \\
\hline $47 \mathrm{X}-3,100-102$ & 440.11 & 455.53 & 2.97 & 20.64 & 0.036 & 0.41 & 8.3 \\
\hline $47 \mathrm{X}-3,110-112$ & 440.21 & 455.63 & 2.56 & 20.77 & $\begin{array}{l}0.028 \\
0.028\end{array}$ & $\begin{array}{l}0.41 \\
0.39\end{array}$ & $\begin{array}{l}0.3 \\
7.6\end{array}$ \\
\hline
\end{tabular}


Table 3 (continued).

\begin{tabular}{|c|c|c|c|c|c|c|c|c|c|c|c|}
\hline $\begin{array}{l}\text { Core, section, } \\
\text { interval }(\mathrm{cm})\end{array}$ & $\begin{array}{l}\text { Depth } \\
\text { (mbsf) }\end{array}$ & $\begin{array}{l}\text { Depth } \\
\text { (mcd) }\end{array}$ & $\begin{array}{l}\mathrm{Al} \\
(\%)\end{array}$ & $\begin{array}{c}\mathrm{Ca} \\
(\%)\end{array}$ & $\begin{array}{c}\mathrm{P} \\
(\%)\end{array}$ & $\begin{array}{c}\mathrm{K} \\
(\%)\end{array}$ & $\begin{array}{l}\mathrm{Si} \\
(\%)\end{array}$ & $\begin{array}{c}\mathrm{Ti} \\
(\%)\end{array}$ & $\begin{array}{c}\mathrm{Ba} \\
(\mathrm{ppm})\end{array}$ & $\begin{array}{c}\mathrm{V} \\
(\mathrm{ppm})\end{array}$ & $\begin{array}{c}\mathrm{Cu} \\
(\mathrm{ppm})\end{array}$ \\
\hline $47 X-3,120-122$ & 440.31 & 455.73 & 2.41 & 21.41 & 0.026 & 0.39 & 7.1 & 0.111 & 698 & 46 & 26 \\
\hline $47 X-3,130-132$ & 440.41 & 455.83 & 2.63 & 21.05 & 0.012 & 0.33 & 7.4 & 0.113 & 837 & 52 & 56 \\
\hline $47 \mathrm{X}-3,140-142$ & 440.51 & 455.93 & 2.96 & 20.75 & 0.032 & 0.27 & 8.3 & 0.119 & 879 & 61 & 41 \\
\hline $47 X-4,0-2$ & 440.61 & 456.03 & 3.40 & 23.87 & 0.032 & 0.45 & 9.8 & 0.145 & 1079 & 55 & 47 \\
\hline $47 X-4,10-12$ & 440.71 & 456.13 & 3.11 & 22.18 & 0.027 & 0.39 & 9.1 & 0.133 & 957 & 57 & 31 \\
\hline $47 X-4,20-22$ & 440.81 & 456.23 & 3.91 & 22.71 & 0.032 & 0.51 & 10.8 & 0.162 & 1067 & 52 & 69 \\
\hline $47 X-4,30-32$ & 440.91 & 456.33 & 3.90 & 20.82 & 0.037 & 0.59 & 10.6 & 0.169 & 1049 & 57 & 60 \\
\hline $47 X-4,40-42$ & 441.01 & 456.43 & 3.53 & 21.25 & 0.040 & 0.47 & 9.8 & 0.155 & 1259 & 66 & 41 \\
\hline $47 X-4,50-52$ & 441.11 & 456.53 & 1.89 & 26.72 & 0.051 & 0.38 & 7.0 & 0.081 & 1117 & 39 & 25 \\
\hline $47 X-4,60-62$ & 441.21 & 456.63 & 1.45 & 27.64 & 0.043 & 0.20 & 6.1 & 0.066 & 1006 & 49 & 34 \\
\hline $47 X-4,70-72$ & 441.31 & 456.73 & 1.74 & 26.56 & 0.026 & 0.21 & 6.1 & 0.076 & 801 & 28 & 29 \\
\hline $47 X-4,80-82$ & 441.41 & 456.83 & 1.95 & 24.37 & 0.027 & 0.29 & 6.3 & 0.085 & 610 & 47 & 24 \\
\hline $47 X-4,90-92$ & 441.51 & 456.93 & 2.54 & 23.09 & 0.030 & 0.37 & 7.5 & 0.117 & 764 & 69 & 52 \\
\hline $47 \mathrm{X}-4,100-102$ & 441.61 & 457.03 & 5.77 & 14.51 & 0.027 & 0.74 & 14.7 & 0.280 & 1377 & 53 & 59 \\
\hline $47 \mathrm{X}-4,110-112$ & 441.71 & 457.13 & 5.15 & 19.70 & 0.042 & 0.73 & 13.0 & 0.262 & 1360 & 86 & 59 \\
\hline $47 \mathrm{X}-4,120-122$ & 441.81 & 457.23 & 3.56 & 22.30 & 0.064 & 0.54 & 9.6 & 0.147 & 1031 & 56 & 37 \\
\hline $47 \mathrm{X}-4,130-132$ & 441.91 & 457.33 & 3.93 & 21.83 & 0.064 & 0.52 & 10.1 & 0.167 & 1127 & 47 & 24 \\
\hline $47 X-4,140-142$ & 442.01 & 457.43 & 4.40 & 22.04 & 0.057 & 0.55 & 11.9 & 0.207 & 1575 & 63 & 70 \\
\hline $47 X-5,0-2$ & 442.11 & 457.53 & 1.98 & 21.98 & 0.044 & 0.25 & 7.5 & 0.092 & 1153 & 36 & 40 \\
\hline $47 X-5,10-12$ & 442.21 & 457.63 & 2.49 & 23.57 & 0.031 & 0.32 & 7.3 & 0.115 & 908 & 41 & 62 \\
\hline $47 X-5,20-22$ & 442.31 & 457.73 & 3.81 & 20.26 & 0.030 & 0.51 & 10.1 & 0.172 & 1072 & 31 & 48 \\
\hline $47 X-5,30-32$ & 442.41 & 457.83 & 4.85 & 16.05 & 0.020 & 0.75 & 13.6 & 0.253 & 1242 & 48 & 46 \\
\hline $47 X-5,40-42$ & 442.51 & 457.93 & 4.94 & 18.59 & 0.047 & 0.77 & 13.6 & 0.213 & 1424 & 88 & 49 \\
\hline $47 X-5,50-52$ & 442.61 & 458.03 & 5.07 & 18.02 & 0.056 & 0.75 & 13.7 & 0.220 & 1321 & 76 & 39 \\
\hline $47 X-5,60-62$ & 442.71 & 458.13 & 3.56 & 21.90 & 0.069 & 0.57 & 10.7 & 0.152 & 1408 & 68 & 40 \\
\hline $47 X-5,70-72$ & 442.81 & 458.23 & 2.25 & 24.68 & 0.064 & 0.38 & 7.9 & 0.097 & 1185 & 57 & 28 \\
\hline $47 X-5,80-82$ & 442.91 & 458.33 & 2.47 & 22.71 & 0.043 & 0.32 & 7.9 & 0.121 & 1228 & 54 & 60 \\
\hline 47X-5, 90-92 & 443.01 & 458.43 & 2.92 & 22.04 & 0.034 & 0.37 & 9.0 & 0.133 & 1132 & 50 & 63 \\
\hline $47 X-5,100-102$ & 443.11 & 458.53 & 4.12 & 19.23 & 0.031 & 0.58 & 12.1 & 0.203 & 1352 & 59 & 73 \\
\hline $47 X-5,110-112$ & 443.21 & 458.63 & 6.50 & 11.97 & 0.037 & 0.94 & 18.8 & 0.374 & 1411 & 91 & 39 \\
\hline $47 X-5,120-122$ & 443.31 & 458.73 & 4.53 & 19.14 & 0.028 & 0.59 & 12.4 & 0.206 & 1462 & 30 & 53 \\
\hline $47 \mathrm{X}-5,130-132$ & 443.41 & 458.83 & 3.61 & 20.08 & 0.054 & 0.47 & 10.5 & 0.161 & 1404 & 35 & 54 \\
\hline $47 X-5,140-142$ & 443.51 & 458.93 & 4.49 & 18.44 & 0.053 & 0.61 & 12.8 & 0.201 & 1780 & 36 & 41 \\
\hline $47 X-6,0-2$ & 443.61 & 459.03 & 3.73 & 17.76 & 0.036 & 0.52 & 10.6 & 0.163 & 1301 & 99 & 74 \\
\hline $47 X-6,10-12$ & 443.71 & 459.13 & 4.33 & 19.48 & 0.039 & 0.51 & 11.6 & 0.188 & 1164 & 74 & 29 \\
\hline $47 X-6,20-22$ & 443.81 & 459.23 & 2.99 & 20.05 & 0.025 & 0.43 & 8.4 & 0.153 & 1082 & 143 & 44 \\
\hline $47 X-6,30-32$ & 443.91 & 459.33 & 3.46 & 22.09 & 0.036 & 0.44 & 10.9 & 0.170 & 1412 & 45 & 43 \\
\hline $47 X-6,40-42$ & 444.01 & 459.43 & 2.01 & 21.30 & 0.031 & 0.27 & 6.9 & 0.092 & 985 & 36 & 67 \\
\hline $47 X-6,50-52$ & 444.11 & 459.53 & 2.01 & 24.67 & 0.035 & 0.25 & 8.0 & 0.090 & 1100 & 55 & 23 \\
\hline $47 X-6,60-62$ & 444.21 & 459.63 & 2.58 & 20.67 & 0.031 & 0.32 & 8.1 & 0.116 & 1078 & 36 & 45 \\
\hline $47 X-6,70-72$ & 444.31 & 459.73 & 2.54 & 20.24 & 0.029 & 0.37 & 8.4 & 0.110 & 1001 & 55 & 36 \\
\hline $47 X-6,80-82$ & 444.41 & 459.83 & 3.40 & 22.35 & 0.037 & 0.46 & 10.5 & 0.141 & 1242 & 79 & 22 \\
\hline 47X-6, 90-92 & 444.51 & 459.93 & 3.93 & 19.65 & 0.034 & 0.50 & 11.3 & 0.170 & 1423 & 48 & 38 \\
\hline $47 \mathrm{X}-6,100-102$ & 444.61 & 460.03 & 4.26 & 21.56 & 0.035 & 0.47 & 11.7 & 0.191 & 1465 & 57 & 130 \\
\hline $47 X-6,110-112$ & 444.71 & 460.13 & 2.69 & 20.45 & 0.031 & 0.36 & 8.0 & 0.127 & 1178 & 50 & 31 \\
\hline $47 \mathrm{X}-6,120-122$ & 444.81 & 460.23 & 2.03 & 25.14 & 0.047 & 0.27 & 7.7 & 0.090 & 1400 & 71 & 55 \\
\hline $47 X-6,130-132$ & 444.91 & 460.33 & 2.21 & 21.25 & 0.034 & 0.27 & 7.3 & 0.103 & 1190 & 53 & 34 \\
\hline $47 X-6,140-142$ & 445.01 & 460.43 & 2.61 & 20.15 & 0.034 & 0.34 & 8.5 & 0.127 & 807 & 93 & 46 \\
\hline $47 X-7,0-2$ & 445.11 & 460.53 & 4.53 & 15.01 & 0.022 & 0.65 & 12.4 & 0.233 & 1020 & 50 & 43 \\
\hline $47 X-7,10-12$ & 445.21 & 460.63 & 4.92 & 15.12 & 0.018 & 0.73 & 13.6 & 0.278 & 1265 & 163 & 60 \\
\hline $47 X-7,20-22$ & 445.31 & 460.73 & 3.91 & 17.84 & 0.023 & 0.46 & 10.8 & 0.213 & 919 & 231 & 34 \\
\hline $47 X-7,30-32$ & 445.41 & 460.83 & 3.11 & 20.21 & 0.035 & 0.36 & 8.8 & 0.138 & 1041 & 45 & 49 \\
\hline 47X-CC, $0-2$ & 445.51 & 460.93 & 2.20 & 24.12 & 0.042 & 0.28 & 7.3 & 0.100 & 996 & 53 & 18 \\
\hline $47 \mathrm{X}-\mathrm{CC}, 10-12$ & 445.61 & 461.03 & 2.13 & 23.33 & 0.035 & 0.31 & 7.3 & 0.090 & 886 & 58 & 18 \\
\hline $47 \mathrm{X}-\mathrm{CC}, 20-22$ & 445.71 & 461.13 & 2.72 & 22.26 & 0.034 & 0.39 & 8.4 & 0.115 & 1078 & 66 & 34 \\
\hline $47 \mathrm{X}-\mathrm{CC}, 30-32$ & 445.81 & 461.23 & 4.01 & 19.78 & 0.032 & 0.51 & 10.9 & 0.170 & 1171 & 68 & 52 \\
\hline
\end{tabular}

Note: Major elements ( $\mathrm{Al}, \mathrm{Ca}, \mathrm{P}, \mathrm{K}, \mathrm{Si}$, and $\mathrm{Ti}$ ) reported as percentage elemental (not oxide) concentrations and minor elements (Ba, $\mathrm{V}$, and $\mathrm{Cu}$ ) reported in ppm.

ratios. Silica concentrations are not especially elevated at these times of supposed high productivity when compared to the values for the Holocene Amazon Fan samples.

The average K/Al value of the latest Miocene samples is considerably higher than that from the older material, but similar to the Holocene fan sediment. This probably indicates an increased proportion of illite in the siliciclastic fraction in the late Miocene. Similarly, average $\mathrm{Ti} / \mathrm{Al}$ values are highest in the same sample run and again match the Holocene results. This may result from the change in clay mineralogy inferred from K/Al data and/or an increased proportion of heavy minerals in the clastic fraction of the sediments. For both elements, these long-term changes in average values are simply explained by the growth of the Amazon Fan in the late Miocene (Castro et al., 1978). This corresponded to a time when the clay minerals of Ceara Rise sediments switched from predominantly kaolinite to illite and the mass accumulation rate of non-carbonate material increased substantially (Curry, Shackleton, Richter, et al., 1995).

\section{Short-Term Changes in Elemental Ratios}

Each run of samples collected at 10-cm intervals allows investigation of the variations in elemental ratios, representing changing sediment composition, over periods of tens of thousands of years. Correlation coefficients between $\mathrm{Ba} / \mathrm{Al}$ and other ratios are cited in the captions to the figures, which show the stratigraphic positions of the ratios. Where sufficient analyses are available, the data have been subjected to spectral analysis based on the Discrete Fourier Transform (methodology in Weedon et al., this volume).

\section{Latest Miocene}

In Core 154-926B-17H, pronounced variations in carbonate content suggested by $\mathrm{Ca} / \mathrm{Al}$ values (Figs. 3, 5A) are also recorded by variations in $\% 550 \mathrm{~nm}$ light reflectance and magnetic susceptibility and relate to regular orbital-climatic forcing (Curry, Shackleton, Richter, et al., 1995). Barium ratios are so similar to the Holocene values that a predominantly detrital source is likely (Fig. 5A). However, there is a moderate correlation with inferred carbonate contents (Fig. 5A), suggesting that a small productivity signal is superimposed on the detrital background. It is possible that these $\mathrm{Ba} / \mathrm{Al}$ values were lowered by dissolution of barites during diagenetic sulfate reduction. The average $\mathrm{Si} / \mathrm{Al}$ and $\mathrm{P} / \mathrm{Al}$ values are also similar to the Holocene siliciclastics ratios. However, these ratios are weakly correlated with $\mathrm{Ba} / \mathrm{Al}$, so a small productivity signal is indicated. 
Table 4. Correlation matrix of elemental concentrations excluding the three Amazon Fan samples $(n=494)$.

\begin{tabular}{|c|c|c|c|c|c|c|}
\hline & $\mathrm{Ca}$ & $\mathrm{P}$ & $\mathrm{K}$ & $\mathrm{Si}$ & $\mathrm{Ti}$ & $\mathrm{Ba}$ \\
\hline $\mathrm{Al}$ & -0.444 & +0.275 & +0.759 & +0.905 & +0.953 & -0.238 \\
\hline $\mathrm{Ca}$ & & $\overline{-0.071}$ & $\overline{-0.324}$ & $\overline{-0.430}$ & $\overline{-0.449}$ & $\overline{+0.085}$ \\
\hline $\mathrm{P}$ & & & $\overline{+0.141}$ & $\overline{+0.306}$ & $\overline{+0.186}$ & +0.113 \\
\hline $\mathrm{K}$ & & & & $\overline{+0.632}$ & $\overline{+0.751}$ & -0.500 \\
\hline $\mathrm{Si}$ & & & & & $\overline{+0.859}$ & $\overline{+0.110}$ \\
\hline $\mathrm{Ti}$ & & & & & & -0.265 \\
\hline
\end{tabular}

Note: $\mathrm{Cu}$ and $\mathrm{V}$ are ommitted as their concentrations are below the limit of quantitation for all samples. Underlined values $=$ the probability that there is no correlation is less than 0.0001 . Bold values $=\mathrm{P}<0.0001$ and $\mathrm{r}$ is greater than 0.5 .

$\mathrm{K} / \mathrm{Al}$ and $\mathrm{Ti} / \mathrm{Al}$ are similar to Holocene values and, though varying erratically, they show some covariation. This similar behavior might indicate short-term, irregular variations in clay mineralogy and/or variations in heavy mineral proportions.

\section{Middle Miocene}

$\mathrm{Ca} / \mathrm{Al}$ results from Core 154-926A-26H (Fig. 5B) show that although inferred carbonate variations are pronounced, $\mathrm{Ba} / \mathrm{Al}$ is very similar to Holocene values and varies erratically. $\mathrm{Si} / \mathrm{Al}$ values are less than the Holocene Amazon Fan results and weakly correlated with $\mathrm{Ba} / \mathrm{Al}$. P/Al values are similar to today and there is no correlation with $\mathrm{Ba} / \mathrm{Al}$. Therefore, there is no evidence for short-term productivity variations. $\mathrm{K} / \mathrm{Al}$ and $\mathrm{Ti} / \mathrm{Al}$ are low and essentially constant, suggesting less illite and/or heavy minerals compared to the late Miocene to Holocene sediments.

\section{Oligocene/Miocene Boundary}

In Cores 154-926B-50X and 51X the average $\mathrm{Ba} / \mathrm{Al}$ values are greater than the Holocene siliciclastic values, suggesting higher average productivity than today (Fig. 6). Biogenic silica was not identified in these cores on board, but small quantities of diatoms, radiolaria, silicoflagellates, and sponge spicules were recorded from the overlying core. Pore-water $\mathrm{SiO}_{2}$ is distinctly elevated at this level in Hole 926B, suggesting that $\mathrm{Si} / \mathrm{Al}$ values have been lowered due to silica dissolution (Curry, Shackleton, Richter, et al., 1995). The weak, although significant, correlation of $\mathrm{Ba} / \mathrm{Al}$ with $\mathrm{Si} / \mathrm{Al}$ and P/Al (Fig. 6 ), suggests that all three relate to productivity variations. Maximum productivity apparently occurred during maximum burial/preservation of carbonate. Spectral analysis reveals regular 1-m variations in all these parameters as well carbonate contents from $\mathrm{Ca} / \mathrm{Al}$ (Fig. 7). The cyclicity corresponds to indirect control by the 40,000-yr (obliquity) orbital-climatic cycles (Weedon et al., this volume). K/Al and $\mathrm{Ti} / \mathrm{Al}$ show the same patterns as found in the middle Miocene.

\section{Mid-Oligocene}

Two data sets have been generated for mid-Oligocene strata, although the time intervals represented are slightly different (Table 1). In Core 154-926B-64X, Ca/Al and hence carbonate contents vary regularly with a wavelength of about $1 \mathrm{~m}$, which is indicative of 40,000-yr cyclicity (Figs 8, 9; Weedon et al., this volume). Ba/Al, Si/ $\mathrm{Al}$, and P/Al mostly exceed Holocene values and show strongly correlated, regular cyclicity (Fig. 8). No biosiliceous material has been described from the core, but high pore-water silica implies dissolution of biogenic material. Again, increased productivity with maxima coincident with maximum carbonate burial/preservation is indicated. $\mathrm{K} / \mathrm{Al}$ and Ti/Al show values similar to results from the middle $\mathrm{Mi}$ ocene and Oligocene/Miocene boundary.

The same patterns as seen in Core 154-926B-64X are also observed in Core 154-929A-47X (Fig. 10). However, while variations
Table 5. Carbonate determinations for Core 154-926B-17H.

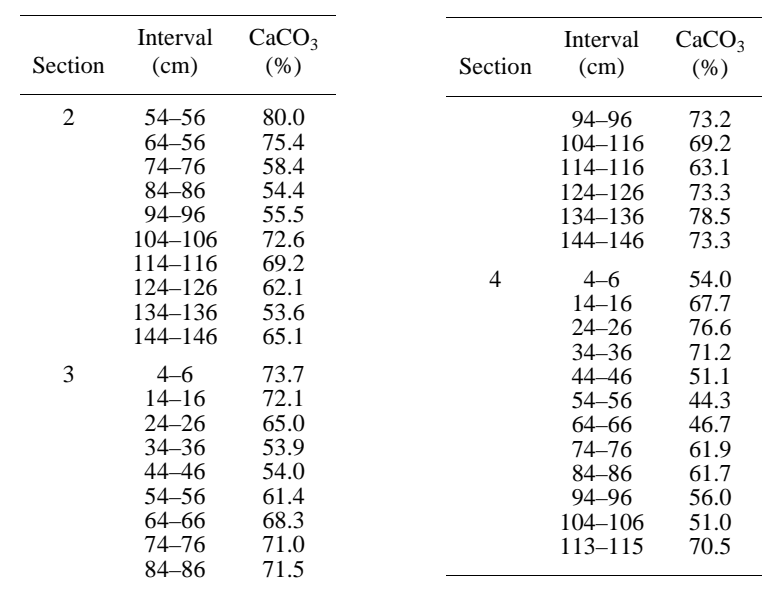

in $\mathrm{Ba} / \mathrm{Al}$ are strongly correlated with $\mathrm{Si} / \mathrm{Al}$ and $\mathrm{P} / \mathrm{Al}$, regular cyclicity cannot be demonstrated rigorously for $\mathrm{Si} / \mathrm{Al}$ (Fig. 11). Nevertheless, pore-water silica concentrations are high, and radiolaria and diatoms form a significant portion of the underlying core. Average Si/Al values are also much higher than any other intervals examined. Thus, regularly varying high productivity is indicated for this deep-water site. Aside from a small interval of relatively high $\mathrm{K} / \mathrm{Al}$ at the top of the core, $\mathrm{K} / \mathrm{Al}$ and $\mathrm{Ti} / \mathrm{Al}$ values are again near-constant and lower than the late Miocene results.

\section{SUMMARY AND CONCLUSIONS}

Despite the potential problems connected with the interpretation of elemental ratios in terms of the controlling factors and mineralogy, a clear pattern emerges from the results. As demonstrated during Leg 154 , illite concentrations in the clastic fraction, as indicated by $\mathrm{K} / \mathrm{Al}$, were low from the mid-Oligocene to the middle Miocene. Increased illite proportions in the clastic fraction occurred in the latest Miocene. The similar behavior of $\mathrm{K} / \mathrm{Al}$ and $\mathrm{Ti} / \mathrm{Al}$ over tens of millions of years, as well as covariation in the late Miocene, might relate to changing clay mineralogy and/or heavy mineral proportions. These changes were probably associated with the growth of the Amazon Fan and an increased flux of siliciclastic material towards the Ceara Rise. This probably coincided with the demise of the South American seaways (Rasanen et al., 1995). If the long-term changes in Ti/Al relate to heavy mineral proportions rather than clay mineralogy, then it might imply a change in sediment provenance that is connected to the derivation of sediment from the Andes rather than more local sources.

Average $\mathrm{Ba} / \mathrm{Al}, \mathrm{Si} / \mathrm{Al}$, and $\mathrm{P} / \mathrm{Al}$ values suggest much higher average productivity in the late early Oligocene and at the Oligocene/Miocene boundary when compared to today. The short-term correlation of $\mathrm{Si} / \mathrm{Al}$ with $\mathrm{Ba} / \mathrm{Al}$ and $\mathrm{P} / \mathrm{Al}$ in the mid-Oligocene and Oligocene/ Miocene boundary shows that the biosiliceous material in the sediment is related to higher productivity and not just preservation. Therefore, the low values of pore-water silica and less common siliceous microfossils in sediments from the early and middle part of the late Oligocene imply that the two periods of increased productivity were separated by a period of lower average productivity. Curry, Shackleton, Richter, et al. (1995), suggested that the changes in deepwater circulation patterns, in both the early Oligocene and latest Oligocene/earliest Miocene, would account for these episodes of increased productivity.

The current data also show that productivity varied on a 40,000yr time scale in the mid-Oligocene and at the Oligocene/Miocene boundary. Maximum productivity occurred during the peak of carbonate burial and minimum carbonate dissolution. Considering the strength of this 40,000-yr (orbital-obliquity) cycle and the lack of ev- 

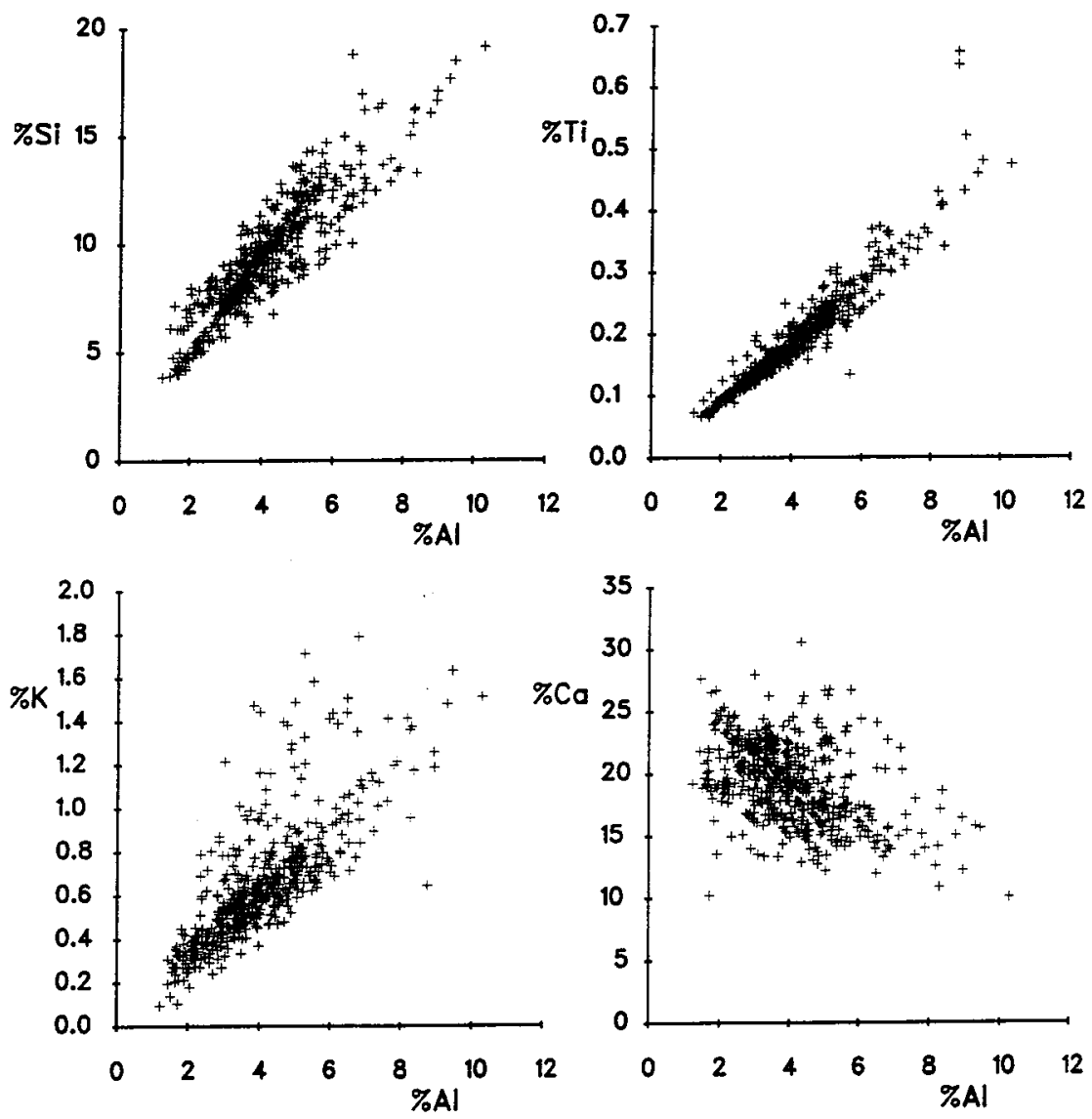

Figure 2. Scatter plots of selected elemental concentrations against aluminum for all the results from the Ceara Rise $(\mathrm{N}=494)$. The strong correlations of $\mathrm{Ti}, \mathrm{K}$, and $\mathrm{Si}$ with $\mathrm{Al}$ (Table 4) indicate that all of these elemental concentrations are related to the proportions of siliciclastic minerals, especially clays. The inverse relationship between $\mathrm{Ca}$ and $\mathrm{Al}$ partly results from varying proportions of calcium carbonate and clay minerals. However, the scatter around this relationship probably results from the presence of calcium in certain clays.

idence for a 20,000-yr component, the mechanism responsible for these short-term variations must have involved high-latitude climatic processes that affected, for example, bottom-water formation.

\section{ACKNOWLEDGMENTS}

Mark Maslin (University College, London) kindly supplied the Leg 155 Amazon Fan samples, and Jan Backman (Stockholm) provided the calcium carbonate determinations. This work was supported by NERC grant GST/02/971. C. Robinson, S. Purdy, and T. Papoloizou (Luton) are thanked for their analytical work. Thanks also to $\mathrm{H}$. Brumsack and M. Delaney for their constructive criticisms.

\section{REFERENCES}

Archer, D., Lyle, M., Rodgers, K., and Froelich, P., 1993. What controls opal preservation in tropical deep-sea sediments? Paleoceanography, 8:7-21.

Arthur, M.A., Jenkyns, H.C., Brumsack, H., and Schlanger, S.O., 1988. Stratigraphy, geochemistry, and paleo-oceanography of organic carbonrich Cretaceous sequences. In Ginsburg, R.N., and Beaudoin, B. (Eds.), Cretaceous Resources, Events and Rhythms. NATO ASI Ser., 304:75119.

Benjamin, M., Johnson, N.M., and Naeser, C.W., 1987. Recent rapid uplift in the Bolivian Andes: evidence from fission-track dating. Geology, 15:680-683.

Brumsack, H.-J., 1986. The inorganic geochemistry of Cretaceous black shales (DSDP Leg 41) in comparison to modern upwelling sediments from the Gulf of California. In Summerhayes, C.P., and Shackleton, N.J. (Eds.), North Atlantic Palaeoceanography. Geol. Soc. London Spec. Publ., 21:447-462.

Brumsack, H.-J., and Gieskes, J.M., 1983. Interstitial water trace-metal chemistry of laminated sediments from the Gulf of California, Mexico. Mar. Chem., 14:89-106.
Castro, J.C., Miura, K., and Estrela-Braga, J.A.E., 1978. Stratigraphic and structural framework of the Foz do Amazonas Basin. Proc. Annu. Offshore Technol. Conf., 3:1843-1847.

Charles, C.D, Froelich, P.N., Zibello, M.A., Mortlock, R.A., and Morley, J.J., 1991. Biogenic opal in southern ocean sediments over the last 450,000 years: implications for surface water chemistry and circulation. Paleoceanography, 6:697-728.

Curry, W., Shackleton, N.J., Richter, C., et al., 1995. Proc. ODP, Init. Repts., 154: College Station, TX (Ocean Drilling Program).

Dymond, J., Suess, E., and Lyle, M., 1992. Barium in deep-sea sediment: a geochemical proxy for paleoproductivity. Paleoceanography, 7:163-182.

Filipelli, G.M., and Delaney, M.L., 1994. The oceanic phosphorus cycle and continental weathering during the Neogene. Paleoceanography, 9:643652.

Flood, R.D., Piper, D.J.W., Klaus, A., et al., 1995. Proc. ODP, Init. Repts., 155: College Station, TX (Ocean Drilling Program).

Gingele, F., and Dahmke, A., 1994. Discrete barium particles and barium as tracers of paleoproductivity in South Atlantic sediments. Paleoceanography, 9:151-168.

Haq, B.U., Hardenbol, J., and Vail, P.R., 1987. Chronology of fluctuating sea levels since the Triassic. Science, 235:1156-1167.

Lea, D.W., and Spero, H.J., 1994. Assessing the reliability of paleochemical tracers: barium uptake in the shells of planktonic foraminifera. Paleoceanography, 9:445-452.

Lyle, M.D., Murray, D.M., Finney, B.P., Dymond, J., Robinson, J.M., and Brookforce, K., 1988. The record of late Pleistocene biogenic sedimentation in the east tropical Pacific Ocean. Paleoceanography, 3:39-59.

Norry, M.J., Dunham, A.C., and Hudson, J.D., 1994. Mineralogy and geochemistry of the Peterborough Member, Oxford Clay Formation, Jurassic, UK: element fractionation during mudrock sedimentation. $J$. Geol. Soc., 151:195-207.

Rasanen, M.E., Linna, A.M., Santo, J.C.R., and Negri, F.R., 1995. Late Miocene tidal deposits in the Amazonian Foreland Basin. Science, 269:386-389.

Schmitz, B., 1989. The $\mathrm{TiO}_{2} / \mathrm{Al}_{2} \mathrm{O}_{3}$ ratio in the Cenozoic Bengal Abyssal Fan sediments and its use as a paleostream energy indicator. Mar. Geol., $76: 195-206$ 
Shimmield, G.B., and Mowbray, S.R., 1991. The inorganic geochemical record of the northwest Arabian Sea: a history of productivity variation over the last 400 k.y. from Sites 722 and 724. In Prell, W.L., Niitsuma, N., et al., Proc. ODP, Sci. Results, 117: College Station, TX (Ocean Drilling Program), 409-429.

Smith, A.G., Smith, D.G., and Funnell, B.M., 1994. Atlas of Mesozoic and Cenozoic Coastlines: Cambridge (Cambridge Univ. Press).

Van Buchem, F.S.P., McCave, I.N., and Weedon, G.P., 1994. Orbitally induced small-scale cyclicity in a siliciclastic epicontinental setting (Lower Lias, Yorkshire, UK). In de Boer, P.L., and Smith, D.G., Orbital Forcing and Cyclic Sequences. Int. Assoc. Sedimentol. Spec. Publ., 19:345-366.

Van Cappellen, P., and Ingall, E.D., 1994. Benthic phosphorus regeneration, net primary production, and ocean anoxia: a model of the coupled marine

biogeochemical cycles of carbon and phosphorus. Paleoceanography, 9:677-692.

Webb, S.D., 1995. Biological implications of the Middle Miocene Amazon seaway. Science, 269:361-362.

Weedon, G.P., and Shimmield, G.B., 1991. Late Pleistocene upwelling and productivity variations in the Northwest Indian Ocean deduced from spectral analyses of geochemical data from Sites 722 and 724. In Prell, W.L., Niitsuma, N., et al., Proc. ODP, Sci. Results, 117: College Station, TX (Ocean Drilling Program), 431-443.

Date of initial receipt: 11 December 1995

Date of acceptance: 14 May 1996

Ms 154SR-129

B

$\% \mathrm{CaCO3}$ 5060708090

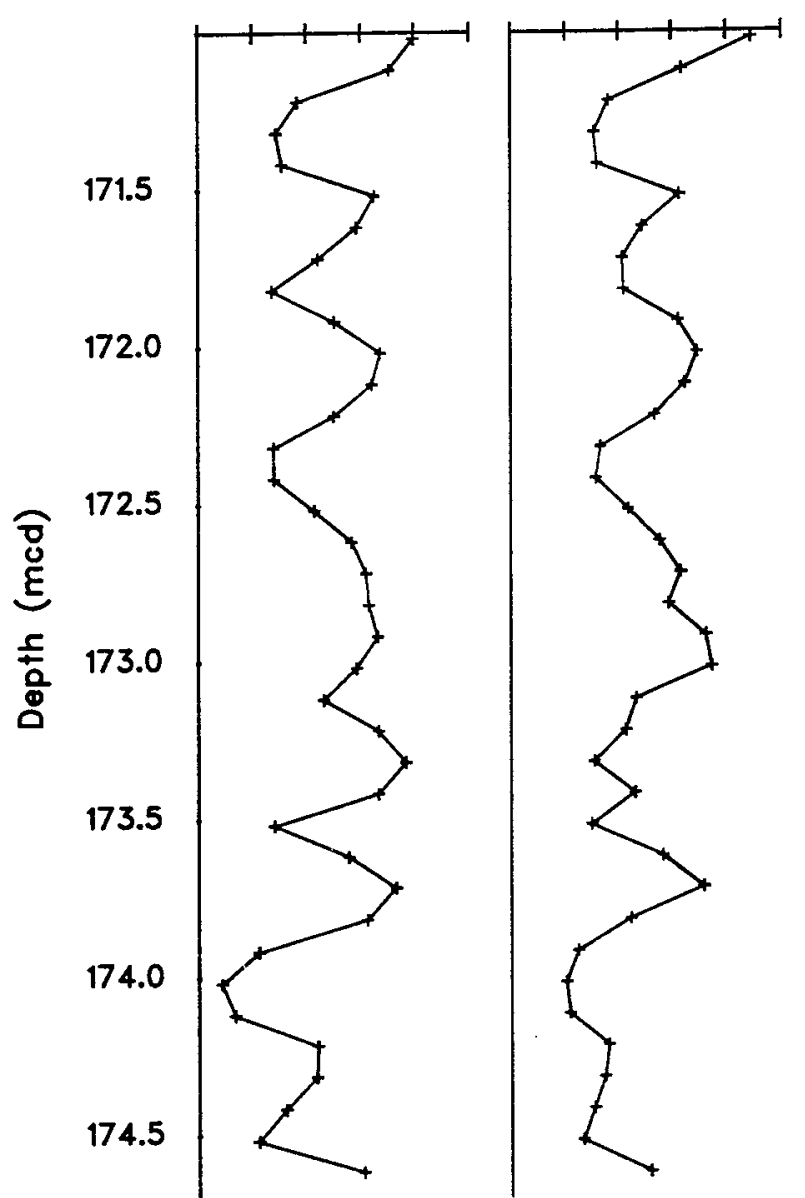

Figure 3. A. Carbonate/non-carbonate vs. Ca/Al values follow an approximately linear relationship in Core 154-926B-17H, as would be expected if most Ca resides in calcite and $\mathrm{Al}$ in clay minerals. B. Plotted stratigraphically, the $\mathrm{Ca} / \mathrm{Al}$ values vary in a similar manner to the measured values of $\mathrm{CaCO}_{3}$ in this core, suggesting that the ratio can be used as a crude proxy for carbonate contents in short stratigraphic intervals. 


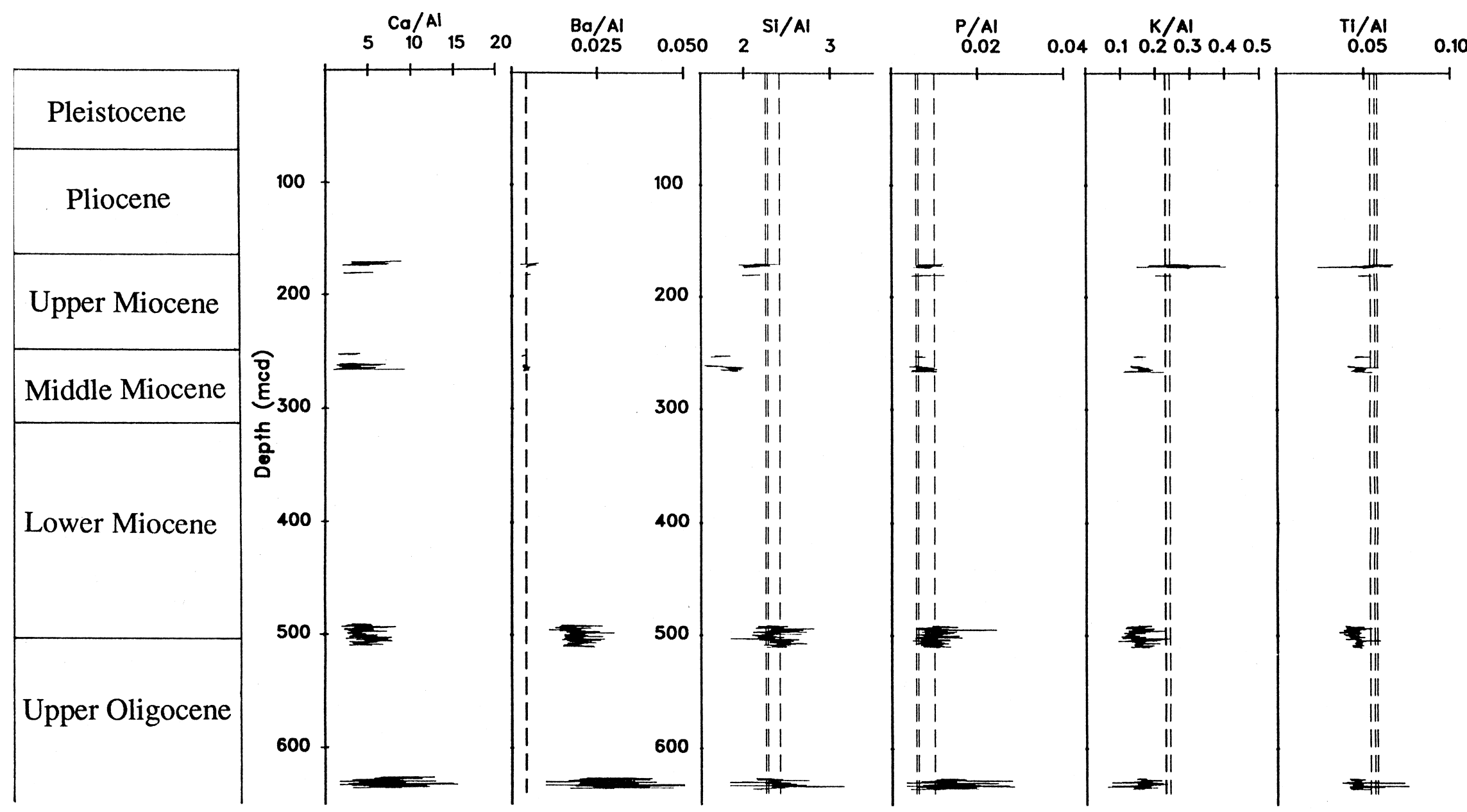

Figure 4. Plot of all the elemental ratios generated for Site 926 (Table 1). Results for Holocene Amazon Fan hemipelagic silty clay from Site 942 (Flood, Piper, Klaus, et al., 1995) are shown as a vertical dashed line. Relatively high $\mathrm{K} / \mathrm{Al}$ in the Holocene and latest Miocene samples suggest relatively elevated illite proportions in the clay fraction. Higher average Ba/Al, Si/Al, and $\mathrm{P} / \mathrm{Al}$ at the Oligocene/Miocene boundary and the mid-Oligocene indicate enhanced productivity compared to the Holocene. Note that Ba/Al values for the uppermost and middle Miocene are very close to the values from the Amazon Fan samples. 

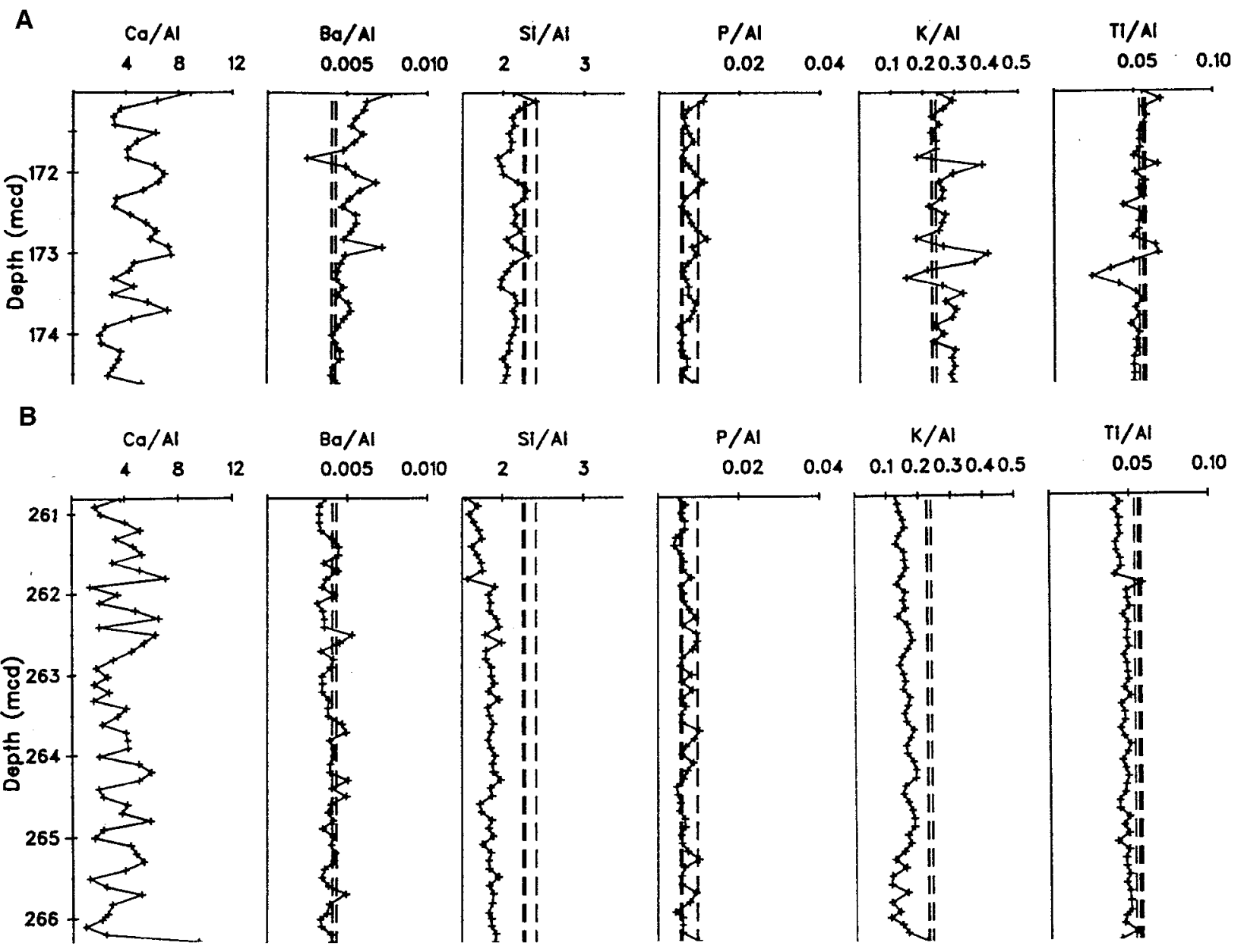

Figure 5. Elemental ratios from sediments deposited (A) in the latest Miocene (Core 154-926B-17H) and (B) in the middle Miocene (Core 154-926A-26H). Results from the Holocene Amazon Fan samples are shown as dashed lines for comparison. For the latest Miocene samples, Ba/Al is weakly correlated with Ca/ $\mathrm{Al}(\mathrm{N}=37, \mathrm{r}=0.63, \mathrm{P}<0.001), \mathrm{P} / \mathrm{Al}(\mathrm{r}=0.59, \mathrm{P}<0.001)$, and $\mathrm{Si} / \mathrm{Al}(\mathrm{r}=0.45, \mathrm{P}<0.01)$. For the middle Miocene samples, $\mathrm{Ba} / \mathrm{Al}$ is weakly correlated with $\mathrm{Ca} /$ $\mathrm{Al}(\mathrm{N}=56, \mathrm{r}=0.35, \mathrm{P}<0.001)$ and $\mathrm{Si} / \mathrm{Al}(\mathrm{r}=0.51, \mathrm{P}<0.0001)$, but not $\mathrm{P} / \mathrm{Al}(\mathrm{r}=0.16$, not significant $)$. 
INORGANIC GEOCHEMICAL COMPOSITION

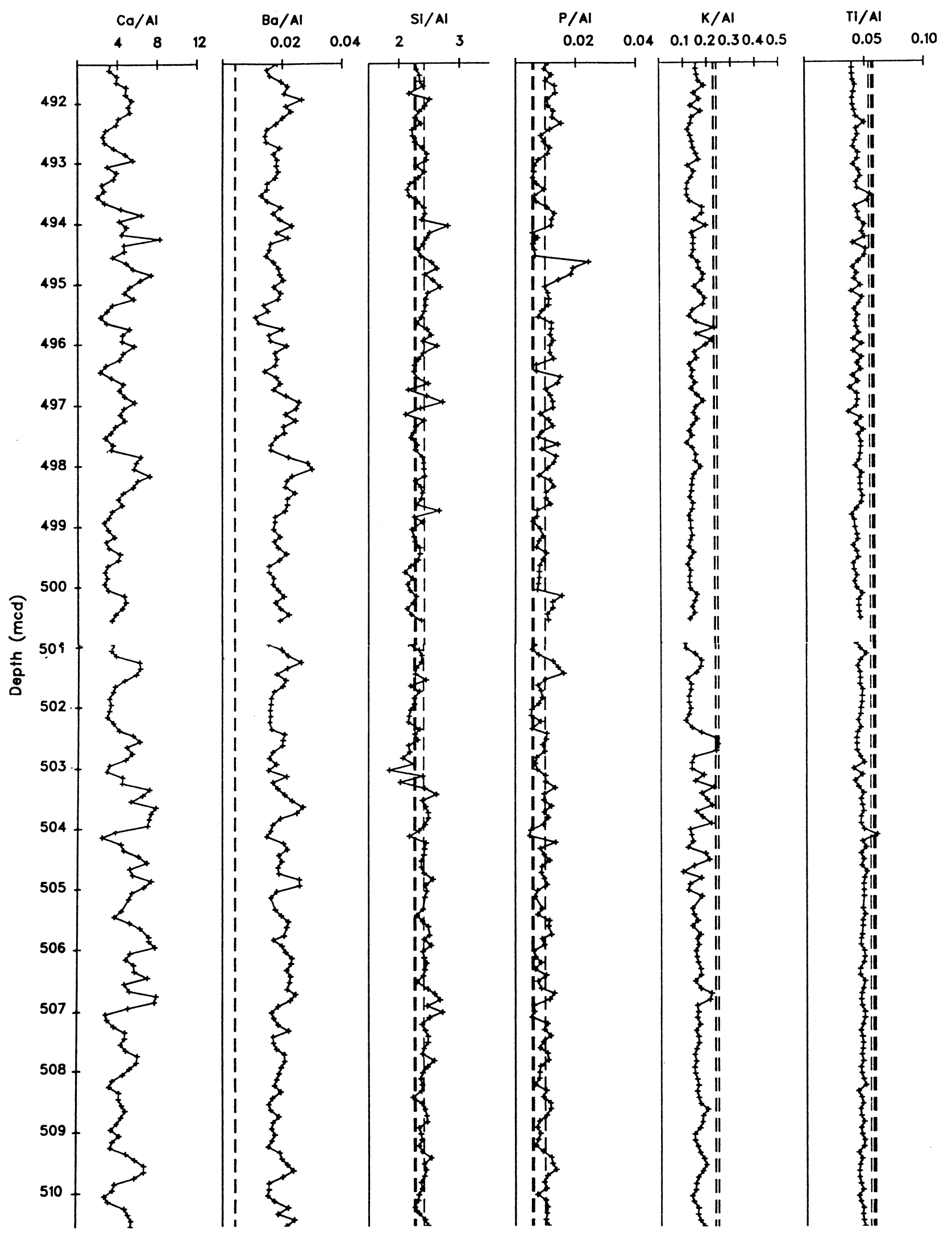

Figure 6. Elemental ratios from samples covering the Oligocene/Miocene boundary from Cores $154-926 \mathrm{~B}-50 \mathrm{X}$ and $51 \mathrm{X}$. Ba/Al is moderately correlated with $\mathrm{Ca} / \mathrm{Al}(\mathrm{N}=189, \mathrm{r}=0.63, \mathrm{P}<0.0001)$, but weakly correlated with $\mathrm{P} / \mathrm{Al}(\mathrm{r}=0.29, \mathrm{P}<0.0001)$ and $\mathrm{Si} / \mathrm{Al}(\mathrm{r}=0.38, \mathrm{P}<0.0001)$. The correlations suggest shortterm variations in productivity occurred at the Oligocene/Miocene boundary over the Ceara Rise. 

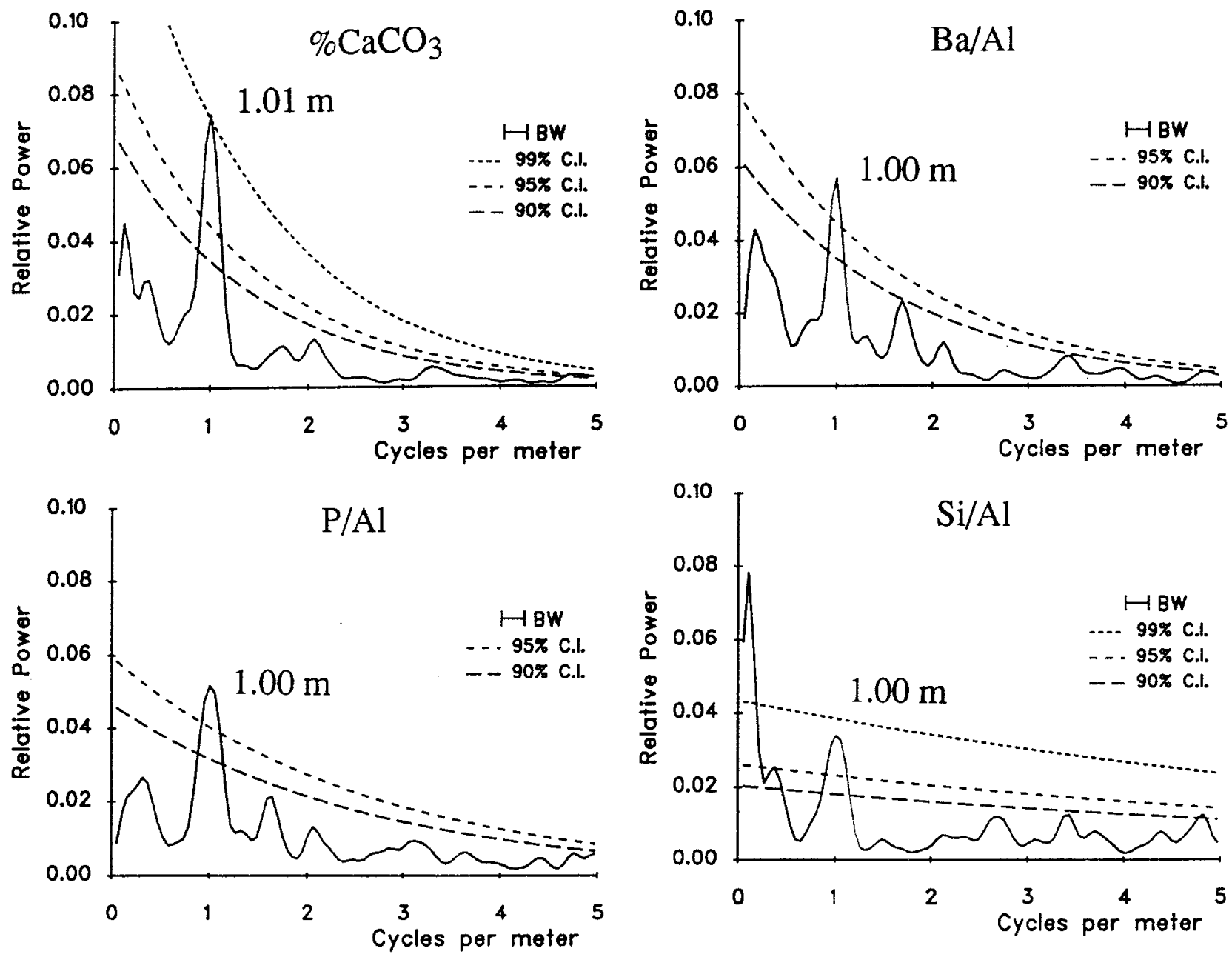

Figure 7. Spectral analysis of certain elemental ratios from the Oligocene/Miocene boundary in Cores 154-926B-50X and 51X. The statistically significant spectral peaks correspond to regular cycles with wavelengths of about $1 \mathrm{~m}$. The cyclicity in Ba/Al, Si/Al, and P/Al results from indirect 40,000-yr orbital-climatic forcing (Weedon et al., this volume). 
INORGANIC GEOCHEMICAL COMPOSITION

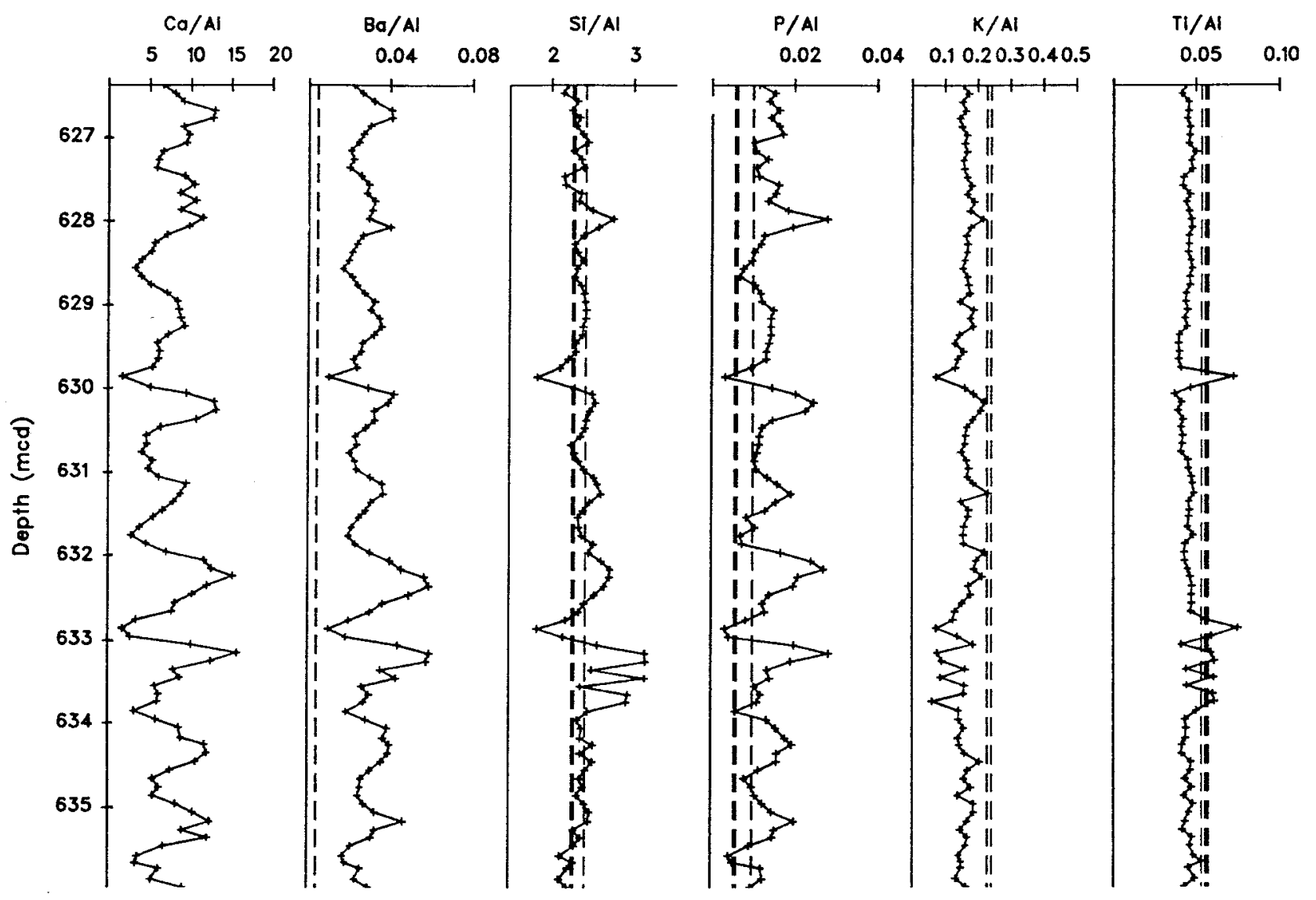

Figure 8. Mid-Oligocene elemental ratios from Core 154-926B-64X. Ba/Al is strongly correlated with $\mathrm{Ca} / \mathrm{Al}(\mathrm{N}=97, \mathrm{r}=0.85), \mathrm{P} / \mathrm{Al}(\mathrm{r}=0.78)$, and $\mathrm{Si} / \mathrm{Al}(\mathrm{r}=$ $0.67 ; \mathrm{P}<0.0001$ for all correlations). These correlations suggest strong productivity variations. 

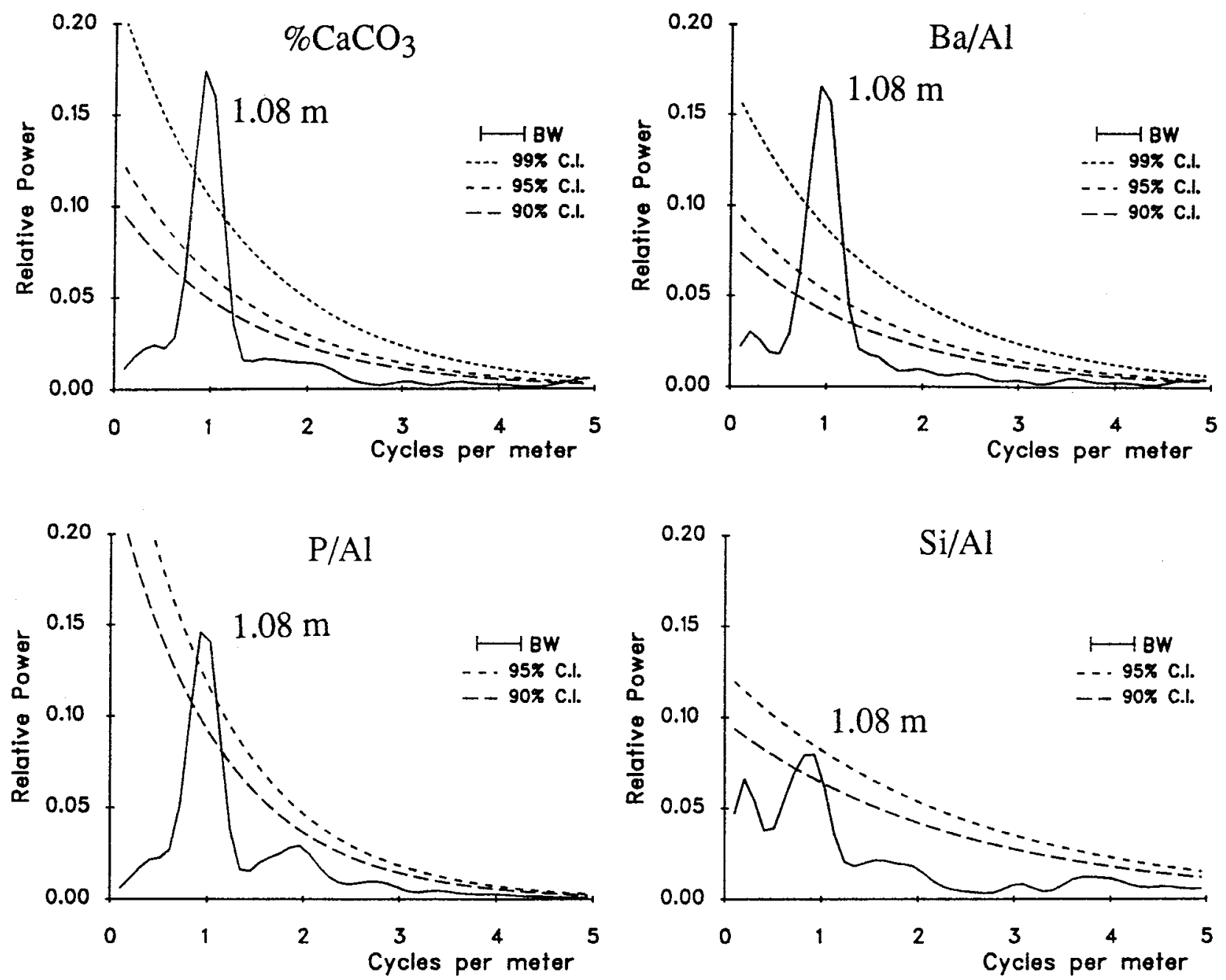

Figure 9. Spectral analysis of mid-Oligocene elemental ratios from Core 154-926B-64X. Regular 40,000-yr cyclicity in productivity is implied by the significant spectral peaks. 

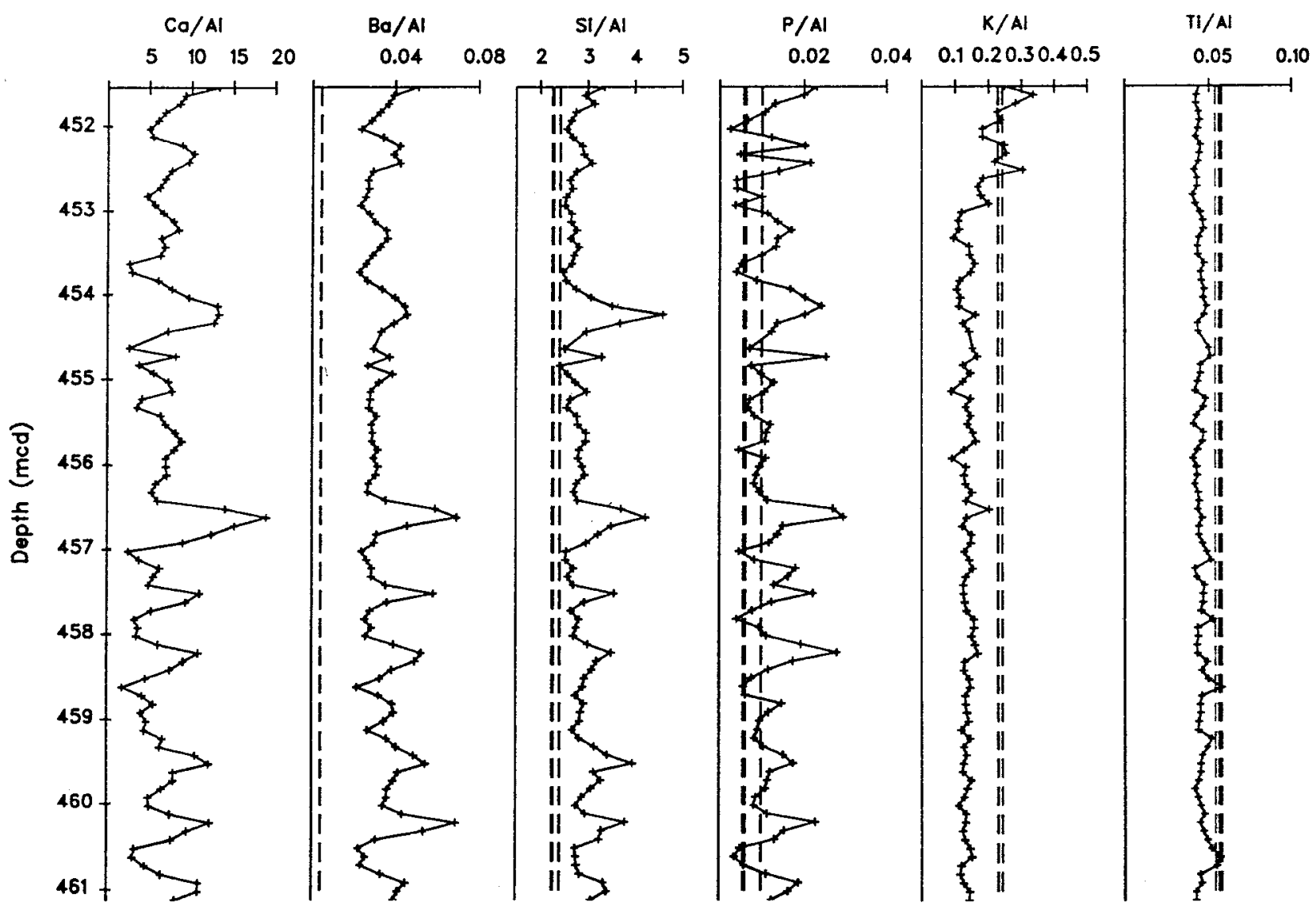

Figure 10. Mid-Oligocene elemental ratios from Core 154-929A-47X. Site 929 is about $60 \mathrm{~km}$ from Site 926 (Fig. 1). There is a strong correlation between Ba/ $\mathrm{Al}$ and $\mathrm{Ca} / \mathrm{Al}(\mathrm{N}=97, \mathrm{r}=0.79), \mathrm{P} / \mathrm{Al}(\mathrm{r}=0.79)$, and $\mathrm{Si} / \mathrm{Al}(\mathrm{r}=0.81 ; \mathrm{P}<0.0001$ for all correlations $)$. These results imply strong productivity variations. Average $\mathrm{Si} / \mathrm{Al}$ values are especially high in this core when compared to the other cores examined, which suggests significantly higher productivity than the Holocene. 

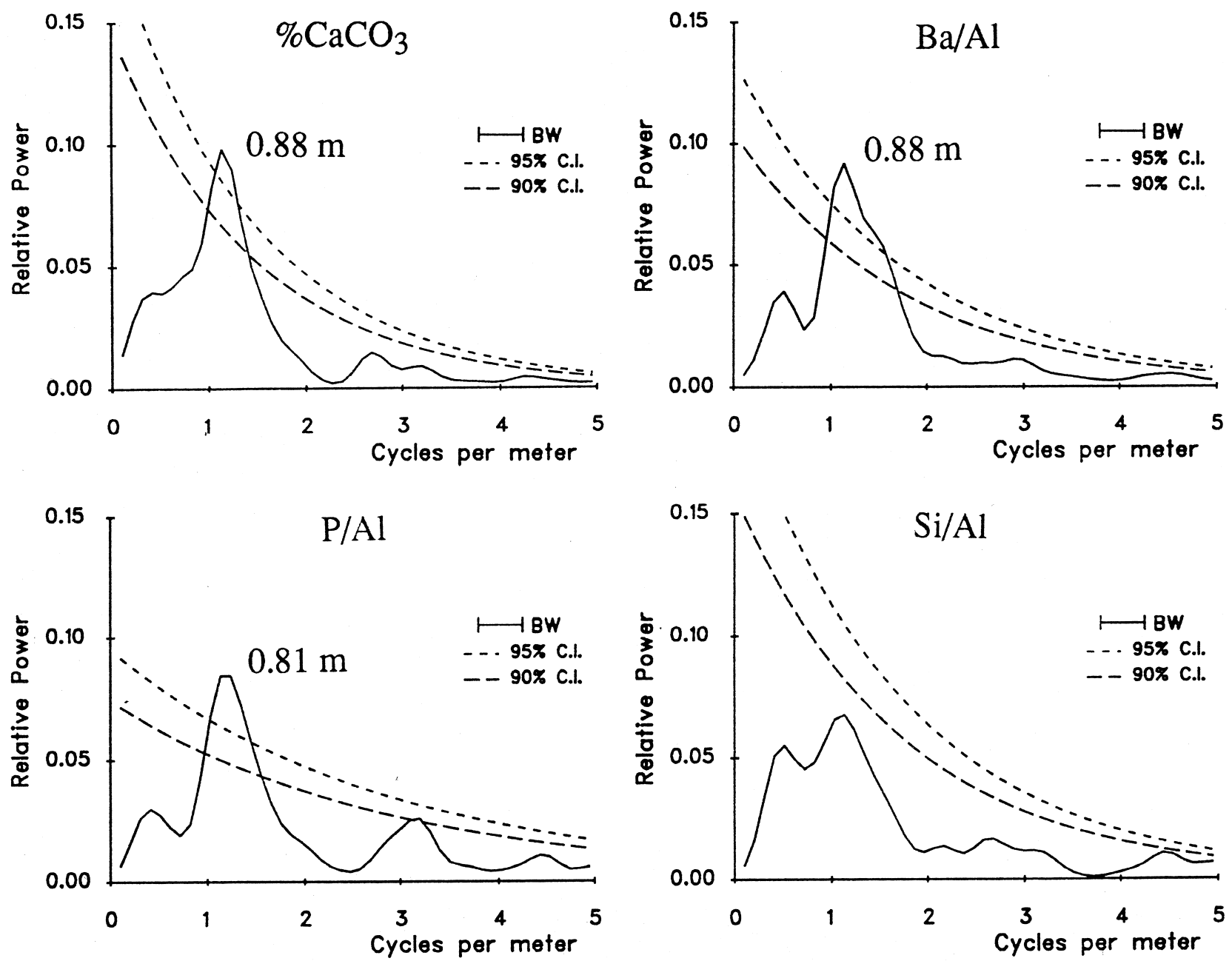

Figure 11. Spectral analysis of mid-Oligocene elemental ratios from Core 154-926B-47X. The spectral peaks indicate regular, approximately $90-\mathrm{cm}$ cyclicity inferred to relate to 40,000 -yr cycles in productivity. 